Pacific

Journal of

Mathematics

SOME RESULTS ON THE GENERIC VANISHING OF KOSZUL COHOMOLOGY VIA DEFORMATION THEORY

JIE WANG

Volume 273 No. 1

January 2015 


\title{
SOME RESULTS ON THE GENERIC VANISHING OF KOSZUL COHOMOLOGY VIA DEFORMATION THEORY
}

\author{
JIE WANG
}

\begin{abstract}
We study the deformation-obstruction theory of Koszul cohomology groups of $g_{d}^{r}$ 's on singular nodal curves. We compute the obstruction classes for Koszul cohomology classes on singular curves to deform to a smooth one. In the case where the obstructions are nontrivial, we obtain some partial results for generic vanishing of Koszul cohomology groups.
\end{abstract}

\section{Introduction}

In this paper, we apply deformation theory to study the syzygies of general curves in $\mathbb{P}^{r}$ with fixed genus and degree. Let $L$ be a basepoint-free $g_{d}^{r}$ on a smooth curve $X$. The Koszul cohomology group $K_{p, q}(X, L)$ is the cohomology of the Koszul complex at the $(p, q)$-spot

$$
\longrightarrow \bigwedge^{p+1} H^{0}(L) \otimes H^{0}\left(X, L^{q-1}\right) \stackrel{d_{p+1, q-1}}{\longrightarrow} \bigwedge^{p} H^{0}(L) \otimes H^{0}\left(X, L^{q}\right) \stackrel{d_{p, q}}{\longrightarrow} H^{0}\left(X, L^{q+1}\right),
$$

where

$$
d_{p, q}\left(v_{1} \wedge \cdots \wedge v_{p} \otimes \sigma\right)=\sum_{i}(-1)^{i} v_{1} \wedge \cdots \wedge \hat{v}_{i} \wedge . . \wedge v_{p} \otimes v_{i} \sigma
$$

The Koszul cohomology groups $K_{p, q}(X, L)$ completely determine the shape of a minimal free resolution of the section ring

$$
R=R(X, L)=\bigoplus_{k \geq 0} H^{0}\left(X, L^{k}\right)
$$

and therefore carry a lot of information about the extrinsic geometry of $X$.

We are interested in Green's question:

Problem 1.1. What is the variational theory of the $K_{p, q}(X, L)$ ? What do they look like for $X$ a general curve and $L$ a general $g_{d}^{r}$ ?

MSC2010: 14H51.

Keywords: Koszul cohomology, general curves, deformation theory, generic vanishing, maximal rank conjecture. 
If $(X, L)$ is general in $\mathcal{G}_{g, d}^{r}$ (in this paper, this means the Brill-Noether number $\rho=g-(r+1)(g-d+r)$ is nonnegative and $(X, L)$ is a general point of the unique component of $\mathcal{G}_{g, d}^{r}$ which dominates $\mathcal{M}_{g}$ ), it is well known that we only have to determine $K_{p, 1}(X, L)$, or equivalently $K_{p-1,2}(X, L)$ for $1 \leq p \leq r-1$ (see Section 2).

Problem 1.1 seems too difficult to answer in full generality. For an arbitrary $g_{d}^{r}$ on a general curve $X$, the simplest cases - determining $K_{1,1}(X, L)$ or $K_{0,2}(X, L)$ are still unknown. The maximal rank conjecture (MRC) [Eisenbud and Harris 1983] predicts that the multiplication map

$$
\operatorname{Sym}^{2} H^{0}(X, L) \stackrel{\mu}{\rightarrow} H^{0}\left(X, L^{2}\right)
$$

is either injective or surjective, that is,

$$
\min \left\{k_{1,1}(X, L), k_{0,2}(X, L)\right\}=0 .
$$

Geometrically, this means that the number of quadrics in $\mathbb{P}^{r}$ containing $X$ is as simple as the Hilbert function of $X \subset \mathbb{P}^{r}$ allows.

There are many partial results about (1-2) using the so-called "méthode d'Horace", originally proposed by Hirschowitz. This amounts to a degeneration argument to a carefully chosen singular curve in projective space and a proof of the statement on such a curve by a delicate inductive argument. We refer to, for instance, [Ballico and Fontanari 2010a; 2010b] for some recent results in this direction.

For higher syzygies, again there are many results (see [Aprodu 2004; Ballico 1996; Ein 1987; Farkas 2009]). One breakthrough result is Voisin's solution [2002; 2005] of the generic Green's conjecture, which solves Problem 1.1 for the case $L=K_{X}$.

For the vanishing of $K_{p, 1}$, there is [Aprodu 2004; 2005], which proved the generic version of the Green-Lazarsfeld gonanity conjecture. This conjecture predicts that, for smooth curve $X$ of gonanity $d$ and $L$ a sufficiently positive line bundle on $X$,

$$
K_{h^{0}(L)-d, 1}(X, L)=0 .
$$

Note that Problem 1.1 does not have any assumption on the positivity of $L$.

It seems that the method of all of the above results amounts to degenerating to special curves, often a carefully chosen singular one, and verifying the statements on these special curves. Given the fact that sometimes such special curves are difficult to find and the inductive arguments can get technical, we would like to take a slightly different point of view. We will consider one-parameter degeneration to the simplest possible singular curve, namely, the union of two smooth curves meeting at a node. Of course, there is no hope of directly verifying the vanishing statements we would like to prove on these curves (see Section 3), but we are able to compute the obstructions for the "extra" Koszul classes of the singular fiber 
to deform to nearby fibers. If one could prove these "extra" Koszul classes are obstructed, we conclude the general fiber has the vanishing property we need. We feel this point of view has a good chance to generalize.

More precisely, let the property $\mathrm{GV}(p)_{g, d}^{r}$ mean that, for general $L^{\prime}=g_{d}^{r}$ on general curve $C$ of genus $g$, we have

$$
\min \left\{k_{p, 1}\left(C, L^{\prime}\right), k_{p-1,2}\left(C, L^{\prime}\right)\right\}=0 .
$$

Problem 1.2. Does $\mathrm{GV}(p)_{g, d}^{r}$ imply $\mathrm{GV}(p)_{g+1, d+1}^{r}$ ?

If this holds, one could set up an inductive argument. At each step $r$ is fixed and $g, d$ go up by 1 , or equivalently, $r$ and $h^{1}$ are fixed, and $g$ goes up by 1 .

In the case $p=1$, the maximal rank conjecture predicts the answer should always be affirmative. For higher syzygies, this is not always the case, but one would like to prove some generic vanishing results for some special $\{g, r, d\}$.

We give a simple condition to guarantee $\mathrm{GV}(p)_{g, d}^{r}$ implies $\mathrm{GV}(p)_{g+1, d+1}^{r}$ from a deformation-theoretic point of view. We study the deformation theory of Koszul cohomology groups on the simplest kind of singular curve $X_{0}$ : the union of a general curve $C$ of genus $g$ and an elliptic curve $E$ meeting at a node $u$. $L_{0}$ is carefully chosen (see Section 3) such that:

(a) $\left(X_{0}, L_{0}\right)$ is smoothable to $L_{t}=g_{d+1}^{r}$ on a smooth curve $X_{t}$ of genus $g+1$.

(b) $\left.L_{0}\right|_{C}=L^{\prime}$ and therefore $\min \left\{k_{p, 1}\left(C,\left.L_{0}\right|_{C}\right), k_{p-1,2}\left(C,\left.L_{0}\right|_{C}\right)\right\}=0$.

(c) $\left.L_{0}\right|_{E}=\mathcal{O}_{E}(v)$ for another general point $v \in E$.

We prove:

Theorem 1.3. Let $C \subset \mathbb{P}^{r}$ be a general curve, $\left|L^{\prime}\right|$ a general $g_{d}^{r}$ on $C$ and $M_{L^{\prime}}$ the kernel bundle defined by the sequence

$$
0 \longrightarrow M_{L^{\prime}} \longrightarrow H^{0}\left(L^{\prime}\right) \otimes \mathcal{O}_{C} \stackrel{\text { ev }}{\longrightarrow} L^{\prime} \longrightarrow 0 .
$$

Then the following hold:

(a) If $K_{p, 1}\left(C, L^{\prime}\right)=0$, then $K_{p, 1}\left(X_{t}, L_{t}\right)=0$.

(b) If $K_{p-1,2}\left(C, L^{\prime}\right)=0$ and

$$
h^{0}\left(C, \wedge^{r-p} M_{L^{\prime}} \otimes K_{C}\right)=h^{0}\left(C, \wedge^{r-p} M_{L^{\prime}} \otimes K_{C}(2 u)\right)
$$

for a general point $u \in C$, then $K_{p-1,2}\left(X_{t}, L_{t}\right)=0$.

In other words, $\mathrm{GV}(p)_{g, d}^{r}$ always implies $\mathrm{GV}(p)_{g+1, d+1}^{r}$ if (1-4) holds.

The upshot is that under such degeneration, we could explicitly compute generators of $K_{p, q}\left(X_{0}, L_{0}\right)$. Unfortunately $\left(X_{0}, L_{0}\right)$ does not satisfy (1-3). However we could compute the obstructions for the "extra" Koszul classes to deform to $K_{p, q}\left(X_{t}, L_{t}\right)$. If every "extra" Koszul class is obstructed, we conclude that (1-3) 
holds for $\left(X_{t}, L_{t}\right)$. Condition (1-4) is a sufficient condition for the "extra" Koszul classes to be obstructed.

In the case $p=1$ (maximal rank conjecture), this sufficient condition turns out to be very geometric.

Theorem 1.4. Let $C \subset \mathbb{P}^{r}$ be a general curve embedded by $\left|L^{\prime}\right|$ a general $g_{d}^{r}$ and suppose one of the following two conditions holds:

(a) $\mu$ in (1-1) is injective.

(b) $\mu$ is surjective and there exists a quadric $Q \in \operatorname{Ker}(\mu)$ containing $C$ but not containing the tangential variety $T C:=\bigcup_{u \in C} T_{u} C$.

Then (MRC) $)_{g+1, d+1}^{r}$ holds as well.

To apply Theorem 1.4 to the maximal rank conjecture, one has to verify a hypothesis in (b) which seems geometrically interesting in its own right. Hopefully there will be some other applications.

Starting from the fact that rational normal curves and canonical curves are projectively normal, we verify hypothesis (b) in some special cases and get some partial results.

Corollary 1.5. Let $(X, L)$ be a general pair in $\mathcal{G}_{g, d}^{r}$ with $h^{1}(L) \leq 1$. Suppose

$$
\begin{array}{ll}
d>\frac{5}{4} g+\frac{9}{4} & \text { if } h^{1}(L)=0, \text { or } \\
d>\frac{5}{4} g+\frac{3}{4} & \text { if } h^{1}(L)=1 ;
\end{array}
$$

then $(X, L)$ is projectively normal.

It is a very well-known result of Green and Lazarsfeld [1986] that any very ample line bundle $L$ on $X$ with

$$
\operatorname{deg}(L) \geq 2 g_{X}+1-2 h^{1}(L)-\operatorname{Cliff}(X)
$$

is projectively normal, and the bound is sharp. Notice that (1-5) implies that $h^{1}(L) \leq 1$.

If $X$ is general,

$$
\operatorname{Cliff}(X)=\left\lfloor\frac{g_{X}-1}{2}\right\rfloor
$$

thus, the Green-Lazarsfeld theorem predicts projective normality for general curves if $d$ is bigger than roughly $3 g / 2$. Corollary 1.5 thus says that if $L$ is also general, we could improve the lower bound of $d$ to roughly $5 \mathrm{~g} / 4$.

The bounds in Corollary 1.5 are weaker than the bounds in [Ballico and Fontanari 2010b].

We could also fix a small $r$ and let $h^{1}$ be arbitrarily large.

Corollary 1.6. The maximal rank conjecture (for quadrics) holds if $r \leq 4$. 
The reason we can get rid of the restriction on the degree of the line bundle for small $r$ is that we can always verify the hypothesis on $T C$ in Theorem 1.4(b) if $r \leq 4$. Thus (MRC) ${ }_{g, d}^{r}$ always implies (MRC) ${ }_{g+1, d+1}^{r}$.

For higher syzygies, we do not expect analogously that $\min \left\{k_{p, 1}, k_{p-1,2}\right\}=0$ for $p \geq 2$. We refer the audience to Section 2 for a counterexample. Nevertheless, we do wish to obtain certain vanishing results or effective upper bounds on $k_{p, q}$.

The difficulty in generalizing the inductive argument to higher syzygies is twofold. First there are relatively few known cases to start the induction with. There is essentially a single known starting series of examples for vanishing of syzygies, namely, Voisin's solution to the generic Green conjecture. Besides Voisin's theorem, Farkas [2006] proved that properties $\mathrm{GV}(2)_{16,21}^{7}$ and $\mathrm{GV}(3)_{22,30}^{10}$ hold. Secondly, for higher syzygies, the sufficient condition for "extra" Koszul classes to be obstructed is not as geometric.

Nevertheless we summarize our results on higher syzygies as follows:

Theorem 1.7. Let $X$ be a general curves of genus $g$ and $L$ a general $g_{d}^{r}$ on $X$. Then:

(a) If $g \geq r+1$, then $K_{p, 1}(X, L)=0$ for $p \geq\lfloor(r+1) / 2\rfloor$.

(b) If $h^{1}(L)=1$ (which implies that $g \geq r+1$ ), then

$$
\begin{array}{ll}
K_{p-1,2}(X, L)=0 & \text { for } 1 \leq p \leq r-\left\lfloor\frac{g}{2}\right\rfloor, \\
k_{p-1,2}(X, L) \leq(g-2 r+2 p-1)\left(\begin{array}{c}
r-1 \\
p-1
\end{array}\right) & \text { for } p>r-\left\lfloor\frac{g}{2}\right\rfloor .
\end{array}
$$

Combining Corollaries 1.5 and 1.7(a), we can determine $k_{p, q}(X, L)$ for $L$ a general $g_{d}^{r}$ with $r \leq 4$.

Corollary 1.8. For a general pair $(X, L)$ in $\mathcal{G}_{g, d}^{r}$ with $r \leq 4, g \geq r+1$, we have

$$
\min \left\{k_{p, 1}(X, L), k_{p-1,2}(X, L)\right\}=0 .
$$

The organization of this paper is as follows. In Section 2, we review some basic facts about Koszul cohomology of general curves. In Section 3, we study the Koszul cohomology of the central fiber $\left(X_{0}, L_{0}\right)$. We explicitly write down the generators of the "extra" Koszul classes in $K_{r-p, 0}\left(X_{0}, L_{0} ; \omega_{X_{0}}\right) \cong K_{p-1,2}\left(X_{0}, L_{0}\right)^{\vee}$. Section 4 contains a computation of the obstructions for these classes to deform, and Section 5 gives a sufficient condition for the obstruction classes to be linearly independent, and a proof of Theorem 1.3. In Section 6, we focus on the $p=1$ case and prove Theorem 1.4 and Corollaries 1.5 and 1.8. Finally, in Section 7, we consider higher syzygies for line bundles with $h^{1}=1$. In some special range of $p$, we are able to prove some vanishing results as in Theorem 1.7. 


\section{Koszul cohomology of general curves}

We first summarize several special properties of Koszul cohomology groups on general curves over $\mathbb{C}$. We refer to [Aprodu and Nagel 2010] and [Eisenbud 1992] for general facts about Koszul cohomology.

Proposition 2.1. Suppose $X$ is a general curve and $L$ is a complete $g_{d}^{r}$ on $X$.

(a) $K_{p, 0}(X, L)=0$ except when $p=0$ and $k_{0,0}(X, L)=1$.

(b) $K_{p, q}(X, L)=0$ for $q \geq 4$.

(c) $K_{p, 3}(X, L)=0$ except when $p=r-1$ and $k_{r-1,3}(X, L)=h^{1}(L)$.

Proof. Statement (a) follows from the definition of Koszul cohomology.

To prove (b) and (c), we use the following facts:

(i) The multiplication map

$$
H^{0}(X, L) \otimes H^{0}\left(X, K_{X} \otimes L^{-1}\right) \longrightarrow H^{0}\left(X, K_{X}\right)
$$

is injective. This is the Gieseker-Petri theorem.

(ii) $H^{0}\left(X, K_{X} \otimes L^{-2}\right)=0$. This is a direct consequence of (i) (see [Arbarello and Cornalba 1981]).

Statement (b) follows from (ii) and the duality theorem of Koszul cohomology (see [Aprodu and Nagel 2010, Section 2.3]):

$$
K_{p, q}(X, L)=K_{r-1-p, 2-q}\left(X, L ; K_{X}\right)^{\vee} .
$$

To prove (c), we first apply (2-1) and note that the Koszul differential $d_{r-1-p,-1}$ factors as

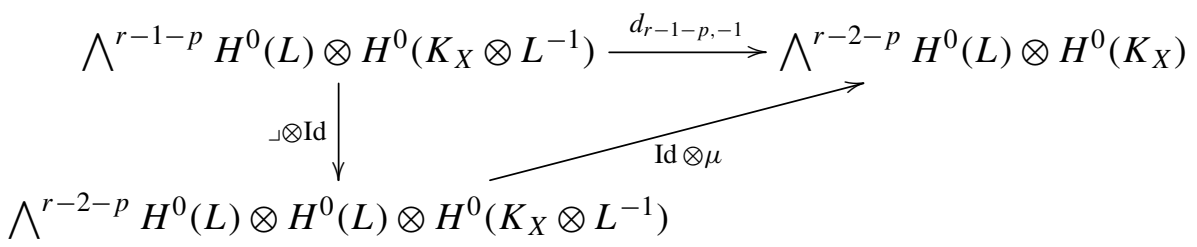

By (i), both $\lrcorner \otimes$ Id and Id $\otimes \mu$ are injective.

As a consequence, we have the following corollary:

Corollary 2.2. Let $X$ be a general curve and $L$ a globally generated $g_{d}^{r}$ with $r \geq 1$.

(a) $L$ is normally generated if and only if the multiplication map

$$
\mu: S^{2} H^{0}(X, L) \longrightarrow H^{0}\left(X, L^{2}\right)
$$

is surjective.

(b) If $L$ is normally generated, the homogeneous ideal $I_{X}$ is generated by quadrics and cubics. 
Proof. The only possible nonzero $K_{0, q}$ for $q \geq 2$ is $K_{0,2}(X, L)=\operatorname{Coker}(\mu)$. If $K_{0,2}(X, L)=0, L$ is normally generated. Since $k_{1, q}$ is the number of minimal generators of $I_{X}$ of degree $q+1$, (b) follows.

Moreover, since taking cohomology does not change the Euler characteristic of the complex, we have for any $1 \leq p \leq r-1$ that

$$
\begin{aligned}
k_{p, 1}(X, L)-k_{p-1,2}(X, L) & \\
= & \sum_{i+j=p+1}(-1)^{j+1} \operatorname{dim}_{\mathbb{C}}\left(\left(\bigwedge^{i} V\right) \otimes H^{0}\left(X, L^{j}\right)\right) \\
& =\left(\begin{array}{c}
r+1 \\
p
\end{array}\right)(g-d+r)-\left(\begin{array}{c}
r+1 \\
p+1
\end{array}\right) g+\left(\begin{array}{c}
r-1 \\
p
\end{array}\right) d+\left(\begin{array}{c}
r \\
p+1
\end{array}\right)(g-1) .
\end{aligned}
$$

Denote this number by $b_{p}(X, L)$, which depends only on $g, r, d, p$. Therefore to determine the Koszul cohomology of $(X, L)$, it suffices to determine either row $q=1$ or $q=2$.

Remark. Based on the maximal rank conjecture, one might expect that analogously

$$
\min \left\{k_{p, 1}(X, L), k_{p-1,2}(X, L)\right\}=0
$$

for general $(X, L)$. But this is not the case. In fact, F. Schreyer proved in his thesis (see [Green 1984, 4.a.2] for more details) that for any curve $X$ of genus $g$, there exists a number $d_{0}$ such that if $\operatorname{deg}(L)=d \geq d_{0}$, then

$$
K_{p, 2}(X, L) \neq 0 \quad \text { if } r-1 \geq p \geq r-g .
$$

On the other hand, it follows from a theorem of Green and Lazarsfeld [1984] (see also [Aprodu and Nagel 2010, Corollary 3.39]) that for $d$ large,

$$
K_{p, 1}(X, L) \neq 0 \quad \text { if } 1 \leq p \leq r-\left\lfloor\frac{g}{2}\right\rfloor-2 .
$$

Thus, for $r-g+1 \leq p \leq r-\lfloor g / 2\rfloor-2$, (2-2) does not hold.

\section{Koszul cohomology of the central fiber}

Let $L^{\prime}$ be a $g_{d}^{r}$ on a smooth curve $C$ of genus $g$ and $X_{0}=C \cup E$ the reducible nodal curve consisting of $C$ and an elliptic curve $E$ meeting at a general point $u$. Let $L_{0}$ be the line bundle on $X_{0}$ such that

$$
\left.L_{0}\right|_{C}=L^{\prime}
$$

and

$$
\left.L_{0}\right|_{E}=\mathcal{O}_{E}(v),
$$

where $v \neq u$. We would like to study the relations between $K_{p, q}\left(C, L^{\prime}\right)$ and $K_{p, q}\left(X_{0}, L_{0}\right)$ in this section. 
First, observe that by construction any (global) section of $L^{\prime}$ on $C$ extends uniquely to a section of $L_{0}$ on $X_{0}$; thus we have a natural isomorphism

$$
\phi: H^{0}\left(C, L^{\prime}\right) \cong H^{0}\left(X_{0}, L_{0}\right) .
$$

Moreover, by the Riemann-Roch theorem, $h^{1}\left(C, L^{\prime}\right)=h^{1}\left(X_{0}, L_{0}\right)$, and there is a natural identification

$$
H^{0}\left(C, K_{C} \otimes L^{\prime-1}\right) \cong H^{0}\left(X_{0}, \omega_{X_{0}} \otimes L_{0}^{-1}\right) .
$$

A first consequence is:

Proposition 3.1. If $K_{p, 1}\left(C, L^{\prime}\right)=0$, then $K_{p, 1}\left(X_{0}, L_{0}\right)=0$.

Proof. Consider the commutative diagram

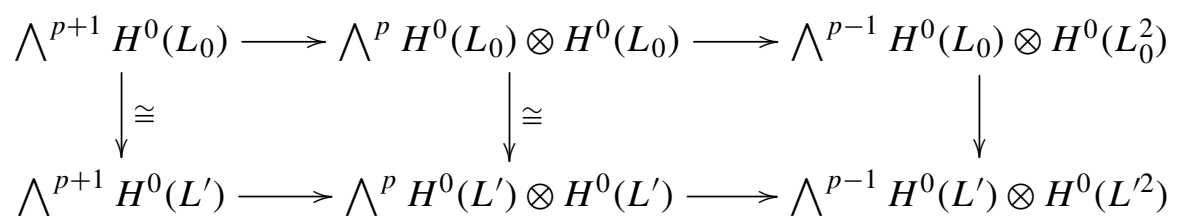

where the vertical arrows are restriction maps to $C$. The hypothesis says that the lower row is exact in the middle. A simple diagram chase gives the conclusion.

The argument in Proposition 3.1 does not generalize to the case $q=2$ because $H^{0}\left(C, L^{\prime 2}\right)$ is not isomorphic to $H^{0}\left(X_{0}, L_{0}^{2}\right)$. Instead we dualize using (2-1),

$$
K_{p-1,2}\left(C, L^{\prime}\right)^{\vee} \cong K_{r-p, 0}\left(C, L^{\prime} ; K_{C}\right),
$$

and compare $K_{r-p, 0}\left(C, L^{\prime} ; K_{C}\right)$ with $K_{r-p, 0}\left(X_{0}, L_{0} ; \omega_{X_{0}}\right)$.

Here, $\omega_{X_{0}}$ is the dualizing sheaf of $X_{0}$. Its restriction to $C$ and $E$ are line bundles $K_{C}(u)$ and $K_{E}(u)$ respectively. A global section of the dualizing sheaf consists of (global) one-forms on $C$ and $E$, viewed as sections of $K_{C}(u)$ and $K_{E}(u)$ respectively which vanish at $u$.

Figure 1 describes the various line bundles in question on $X_{0}$ and their restrictions to each component. The S-shaped curve is $C$ and the straight line is $E$.

Choose a basis $\left\{\omega_{0}, \ldots, \omega_{g-1}\right\}$ of $H^{0}\left(C, K_{C}\right)$ and a basis $\left\{\omega_{g}\right\}$ of $H^{0}\left(E, K_{E}\right)$. For $0 \leq i \leq g-1$, we will think of $\omega_{i}$ as a section in $H^{0}\left(K_{C}(u)\right)$ which vanishes on $u$, and then extend it over $E$ by the zero section. Such a section belongs to $H^{0}\left(\omega_{X_{0}}\right)$, and we still denote it by $\omega_{i}$. Similarly, we obtain $\omega_{g} \in H^{0}\left(\omega_{X_{0}}\right)$ with $\left.\omega_{g}\right|_{C}=0$.

In this way, we obtain a natural identification

$\psi: H^{0}\left(C, K_{C}\right) \oplus H^{0}\left(E, K_{E}\right) \cong H^{0}\left(C, K_{C}(u)\right) \oplus H^{0}\left(E, K_{E}(u)\right) \cong H^{0}\left(X_{0}, \omega_{X_{0}}\right)$ and

$$
H^{0}\left(X_{0}, \omega_{X_{0}}\right)=\operatorname{span}\left\{\omega_{i} \mid i=1, \ldots, g\right\}
$$




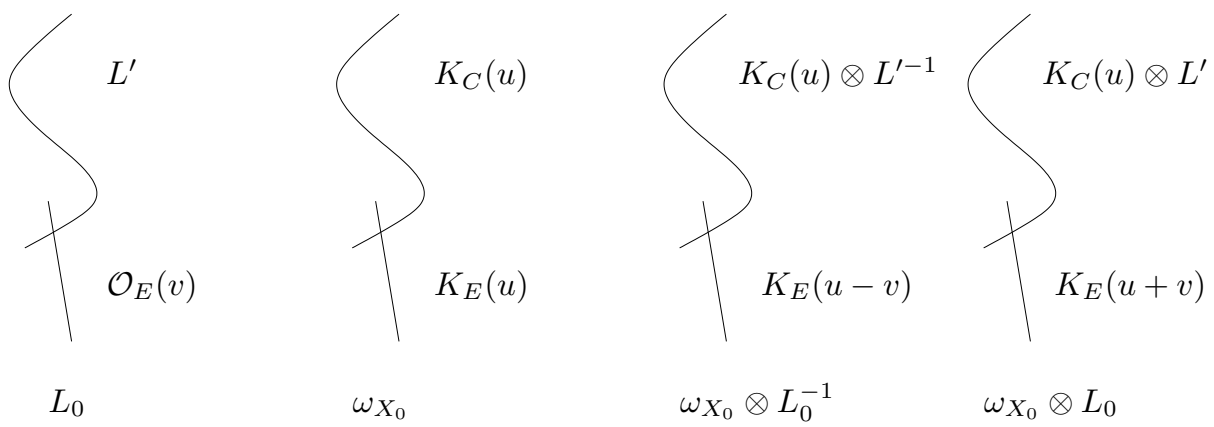

Figure 1. The line bundles on the central fiber.

Notice also that every section in $H^{0}\left(X_{0}, \omega_{X_{0}}\right)$ vanishes at $u$.

Now suppose $K_{r-p, 0}\left(C, L^{\prime} ; K_{C}\right)=0$. We want to show that $K_{r-p, 0}\left(X_{0}, L_{0} ; \omega_{X_{0}}\right)$ can be generated by pure tensors in

$$
\bigwedge^{r-p} H^{0}\left(X_{0}, L_{0}\right) \otimes H^{0}\left(X_{0}, \omega_{X_{0}}\right)
$$

To this end, consider the commutative diagram

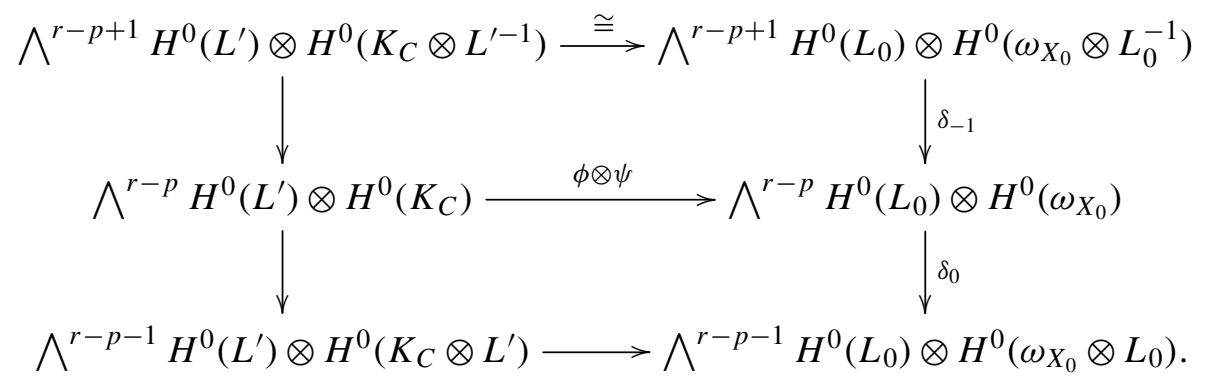

The top horizontal arrow is an isomorphism since any section in $H^{0}\left(X_{0}, \omega_{X_{0}} \otimes L_{0}^{-1}\right)$ restricts to zero on the $E$ component.

Now let $\left\{\sigma_{0}, \ldots, \sigma_{r}\right\}$ be a basis of $H^{0}\left(C, L^{\prime}\right)$. Extend each $\sigma_{k}$ uniquely to $X_{0}$ to form a basis of $H^{0}\left(X_{0}, L_{0}\right)$, still denoting them by $\sigma_{k}$.

We can write any element in $\operatorname{Ker}\left(\delta_{0}\right)$ as

$\sum_{\substack{k_{1}, \ldots, k_{r-p} \\ j \leq g-1}} \alpha_{k_{1}, \ldots, k_{r-p}, j} \sigma_{k_{1}} \wedge \cdots \wedge \sigma_{k_{r-p}} \otimes \omega_{j}+\sum_{k_{1}, \ldots, k_{r-p}} \beta_{k_{1}, \ldots, k_{r-p}} \sigma_{k_{1}} \wedge \cdots \wedge \sigma_{k_{r-p}} \otimes \omega_{g}$

Since the image under $\delta_{0}$ of the second term $\beta$ restricts to 0 on $C$ (since $\omega_{g}$ does), so does the image of the first term $\alpha$. By our assumption, the left column of the above diagram is exact in the middle and therefore $\alpha \in \operatorname{Im}\left(\delta_{-1}\right)$. 
We conclude that

$$
\sum_{k_{1}, \ldots, k_{r-p}} \beta_{k_{1}, \ldots, k_{r-p}} \sigma_{k_{1}} \wedge \cdots \wedge \sigma_{k_{r-p}} \otimes \omega_{g} \in \operatorname{Ker}\left(\delta_{0}\right),
$$

and this can happen only if

$$
\sum_{k_{1}, \ldots, k_{r-p}} \beta_{k_{1}, \ldots, k_{r-p}} \sigma_{k_{1}} \wedge \cdots \wedge \sigma_{k_{r-p}} \in \wedge^{r-p} V
$$

where $V \subset H^{0}\left(X_{0}, L_{0}\right)$ is the codimension-one subspace consisting of sections which restrict to zero on $E$. Also, it is easy to see that a basis of

$$
\bigwedge^{r-p} V \otimes \mathbb{C} \cdot \omega_{g}
$$

is linearly independent even modulo $\operatorname{Im}\left(\delta_{-1}\right)$.

We have proved:

Lemma 3.2. If $K_{r-p, 0}\left(C, L^{\prime} ; K_{C}\right)=0$, we have an isomorphism

$$
K_{r-p, 0}\left(X_{0}, L_{0} ; \omega_{X_{0}}\right) \stackrel{\cong}{\longrightarrow} \bigwedge^{r-p} V \otimes \mathbb{C} \cdot \omega_{g} .
$$

\section{Infinitesimal calculations}

In this section, we carry out the computation of first-order obstructions described in the introduction. We will use the deformation theory of complexes, which was developed in [Green and Lazarsfeld 1987]. The general set-up is as below.

Let $S$ be a smooth variety and $F^{\bullet}$ a bounded complex of locally free sheaves on $S$ :

$$
\cdots \longrightarrow F^{p+1} \stackrel{d_{p+1}}{\longrightarrow} F^{p} \stackrel{d_{p}}{\longrightarrow} F^{p-1} \longrightarrow \cdots .
$$

Given a point $t \in S$, denote by $F^{\bullet}(t)$ the complex of vector spaces at $t$ determined by the fibers of $F^{\bullet}$; that is,

$$
F^{\bullet}(t)=F^{\bullet} \otimes \mathbb{C}(t),
$$

where $\mathbb{C}(t)$ is the residue field of $S$ at $t$.

The deformation theory of $H^{i}\left(F^{\bullet}(t)\right)$ as $t$ moves near $0 \in S$ is controlled by the derivative complex, which associates to a tangent vector $v \in T_{0} S$ a complex

$$
\cdots \longrightarrow H^{p+1}\left(F^{\bullet}(0)\right) \stackrel{D_{v}\left(d_{p+1}\right)}{\longrightarrow} H^{p}\left(F^{\bullet}(0)\right) \stackrel{D_{v}\left(d_{p}\right)}{\longrightarrow} H^{p-1}\left(F^{\bullet}(0)\right) \longrightarrow \cdots .
$$

A (co)homology class $[c] \in H^{p}\left(F^{\bullet}(0)\right)$ deforms to first order along $v$ if and only if $D_{v}\left(d_{p}\right)([c])=0 \in H^{p-1}\left(F^{\bullet}(0)\right)$.

To describe the $D_{v}\left(d_{p}\right)$, recall that a tangent vector $v \in T_{0} S$ corresponds to an embedding of the dual numbers $D$ into $S$, so one gets a short exact sequence

$$
0 \longrightarrow \mathbb{C}(0) \longrightarrow D \longrightarrow \mathbb{C}(0) \longrightarrow 0
$$


Tensoring the sequence with $F^{\bullet}$ yields a short exact sequence of complexes, which in turn gives rise to connecting homomorphisms

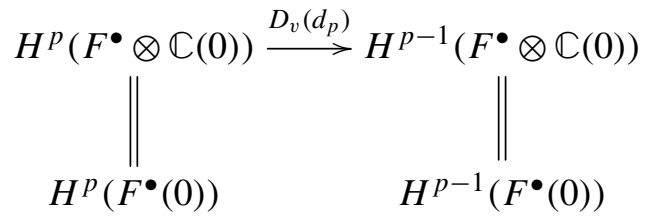

One checks that $D_{v}\left(d_{p}\right) \circ D_{v}\left(d_{p+1}\right)=0$.

Now let $\left(X_{0}, L_{0}\right)$ be the pair constructed in the previous section. We will further assume that both $\left(C, L^{\prime}\right)$ and the crossing point $u$ are general. $\left(X_{0}, L_{0}\right)$ determines a limit linear series in the sense of [Eisenbud and Harris 1986]. By counting Brill-Noether numbers, it is easy to see this limit linear series is deformable to general pairs $\left(X_{t}, L_{t}\right)$. Let $\mathcal{L} \rightarrow \mathcal{X} \rightarrow \Delta$ be the total space of a one-parameter family of general pairs $\left(X_{t}, L_{t}\right) \in \mathcal{G}_{g+1, d+1}^{r}$ degenerating to $\left(X_{0}, L_{0}\right)$. We will apply the deformation theory described above to the Koszul complex computing $K_{r-p, 0}\left(X_{t}, L_{t} ; \omega_{X_{t}}\right)$ :

$$
\begin{aligned}
\bigwedge^{r-p+1} H^{0}\left(L_{t}\right) \otimes H^{0}\left(\omega_{X_{t}} \otimes L_{t}^{-1}\right) & \longrightarrow \Lambda^{r-p} H^{0}\left(L_{t}\right) \otimes H^{0}\left(\omega_{X_{t}}\right) \\
& \stackrel{\delta_{t}}{\longrightarrow} \bigwedge^{r-p-1} H^{0}\left(L_{t}\right) \otimes H^{0}\left(\omega_{X_{t}} \otimes L_{t}\right) .
\end{aligned}
$$

By the Gieseker-Petri theorem, the left arrow is injective for all $t$ (even at time zero), so $k_{r-p, 0}\left(X_{t}, L_{t} ; \omega_{X_{t}}\right)$ can only increase at $t=0$ if $\operatorname{Ker}\left(\delta_{t}\right)$ does. We would like to compute the derivative of $\delta_{t}$ at $t=0$ :

$$
K_{r-p, 0}\left(X_{0}, L_{0} ; \omega_{X_{0}}\right) \stackrel{\left.D\left(\delta_{t}\right)\right|_{t=0}}{\longrightarrow} K_{r-p-1,1}\left(X_{0}, L_{0} ; \omega_{X_{0}}\right) .
$$

To illustrate the idea, let us first take a look at the simpler case when $p=r-1$. (On the other hand, the main case we are interested in is the case $p=1$.) The general case is just notationally more complicated. In this special case, the Koszul differential $\delta_{t}$ becomes the multiplication map $\mu_{t}$

$$
\bigwedge^{2} H^{0}\left(L_{t}\right) \otimes H^{0}\left(\omega_{X_{t}} \otimes L_{t}^{-1}\right) \hookrightarrow H^{0}\left(L_{t}\right) \otimes H^{0}\left(\omega_{X_{t}}\right) \stackrel{\mu_{t}}{\longrightarrow} H^{0}\left(\omega_{X_{t}} \otimes L_{t}\right),
$$

and the derivative map is

$$
K_{1,0}\left(X_{0}, L_{0} ; \omega_{X_{0}}\right) \stackrel{\left.D\left(\mu_{t}\right)\right|_{t=0}}{\longrightarrow} K_{0,1}\left(X_{0}, L_{0} ; \omega_{X_{0}}\right) .
$$

For simplicity, denote $\omega_{g} \in H^{0}\left(X_{0}, \omega_{X_{0}}\right)$ by $\omega$. If $K_{1,0}\left(C, L^{\prime}, K_{C}\right)=0$, then by Lemma 3.2 we have

$$
K_{1,0}\left(X_{0}, L_{0} ; \omega_{X_{0}}\right) \stackrel{\cong}{\longrightarrow} V \otimes \mathbb{C} \cdot \omega \subset V \otimes H^{0}\left(X_{0}, \omega_{X_{0}}\right) .
$$

Remark. Even if $K_{1,0}\left(C, L^{\prime}, K_{C}\right) \neq 0$, we nevertheless have the containment $V \otimes \mathbb{C} \cdot \omega \subset K_{1,0}\left(X_{0}, L_{0} ; \omega_{X_{0}}\right)$. 


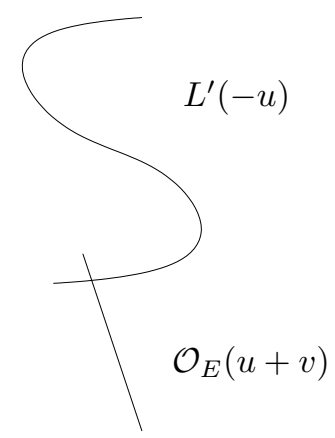

$M$

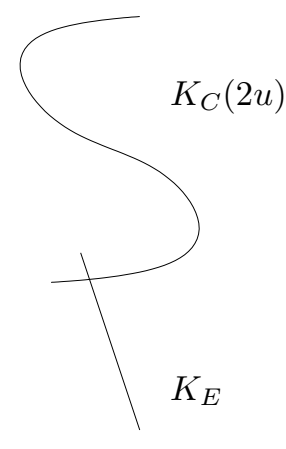

$N$

Figure 2. The twisted line bundles on the central fiber.

So, let $\sigma \in V$. By the description of the derivative complex at the beginning of this section, to compute

$$
\left.D\left(\mu_{t}\right)\right|_{t=0}(\sigma \otimes \omega),
$$

we have to lift $\sigma \otimes \omega$ to first order in $t$, apply the Koszul differential $\mu_{t}$ to the lifting, then restrict the outcome divided by $t$ to $X_{0}$.

So, let $\tilde{\sigma}, \tilde{\omega}$ be sections of $\mathcal{L}$ and $\omega_{\mathcal{X} / \Delta}$ extending $\sigma$ and $\omega$, respectively.

Since $\tilde{\sigma}$ vanishes on $E$ and $\tilde{\omega}$ vanishes on $C$, we can write

$$
\tilde{\sigma}=\tilde{\sigma}^{\prime} s_{E}
$$

and

$$
\tilde{\omega}=\tilde{\omega}^{\prime} s_{C},
$$

where $s_{E}$ and $s_{C}$ are sections of $\mathcal{O}_{\mathcal{X}}(E)$ and $\mathcal{O}_{\mathcal{X}}(C)$ vanishing precisely on $E$ and $C$, respectively, and $\tilde{\sigma}^{\prime}$ and $\tilde{\omega}^{\prime}$ are global sections of

$$
M:=\left.\left.\mathcal{L}(-E)\right|_{X_{0}} \cong \mathcal{L}(C)\right|_{X_{0}} \quad \text { and } \quad N:=\left.\left.\omega_{\mathcal{X} / \Delta}(-C)\right|_{X_{0}} \cong \omega_{\mathcal{X} / \Delta}(E)\right|_{X_{0}},
$$

respectively. Notice that tensoring $\mathcal{L}$ by $\mathcal{O}_{\mathcal{X}}(-E)$ will increase the degree by 1 on the $E$ component and decrease the degree by 1 on the $C$ component. The line bundles $M$ and $N$ are described in Figure 2. Notice that $M \otimes N \cong \omega_{X_{0}} \otimes L_{0}$.

By the construction of the derivative complex,

(4-4) $\left.D\left(\mu_{t}\right)\right|_{t=0}(\sigma \otimes \omega)=\left.\frac{\tilde{\sigma} \cdot \tilde{\omega}}{t}\right|_{X_{0}}=\left.\frac{\left(\tilde{\sigma}^{\prime} s_{E}\right) \cdot\left(\tilde{\omega}^{\prime} s_{C}\right)}{t}\right|_{X_{0}}=\left.\left(\tilde{\sigma}^{\prime} \tilde{\omega}^{\prime}\right)\right|_{X_{0}} \bmod \operatorname{Im} \mu_{0}$.

The general case is just notationally more complicated.

Let

$$
\sigma_{i_{1}} \wedge \cdots \wedge \sigma_{i_{r-p}} \otimes \omega \in \bigwedge^{r-p} V \otimes H^{0}\left(E, K_{E}\right)
$$


We will compute its image under $\left.D\left(\delta_{t}\right)\right|_{t=0}$. Similarly to the simpler case, we have to lift $\sigma_{i_{1}} \wedge \cdots \wedge \sigma_{i_{r-p}} \otimes \omega$ to first order, apply the Koszul differential $\delta_{t}$ to the lifting, then restrict the outcome divided by $t$ to $X_{0}$.

To this end, let $\tilde{\sigma}_{i_{k}}, \tilde{\omega}$ be sections of $\mathcal{L}$ and $\omega_{\mathcal{X} / \Delta}$ extending $\sigma_{i_{k}}$ and $\omega$, respectively. Since $\tilde{\sigma}_{i_{k}}$ vanishes on $E$ and $\tilde{\omega}$ vanishes on $C$, we can write

$$
\tilde{\sigma}_{i_{k}}=\tilde{\sigma}_{i_{k}}^{\prime} s_{E}
$$

and

$$
\tilde{\omega}=\tilde{\omega}^{\prime} s_{C},
$$

as before.

We compute

$$
\begin{aligned}
\left.D\left(\delta_{t}\right)\right|_{t=0} & \left(\sigma_{i_{1}} \wedge \cdots \wedge \sigma_{i_{r-p}} \otimes \omega\right) \\
& =\left.\frac{\delta\left(\tilde{\sigma}_{i_{1}} \wedge \cdots \wedge \tilde{\sigma}_{i_{r-p}} \otimes \tilde{\omega}\right)}{t}\right|_{X_{0}} \\
& =\left.\sum_{k=1}^{r-p}(-1)^{k} \frac{\tilde{\sigma}_{i_{1}} \wedge \cdots \wedge \hat{\tilde{\sigma}}_{i_{k}} \wedge \cdots \wedge \tilde{\sigma}_{i_{r-p}} \otimes\left(\tilde{\sigma}_{i_{k}}^{\prime} s_{E}\right)\left(\tilde{\omega}^{\prime} s_{C}\right)}{t}\right|_{X_{0}} \\
& =\left.\sum_{k=1}^{r-p}(-1)^{k} \sigma_{i_{1}} \wedge \cdots \wedge \hat{\sigma}_{i_{k}} \wedge \cdots \wedge \sigma_{i_{r-p}} \otimes\left(\tilde{\sigma}_{i_{k}}^{\prime} \tilde{\omega}^{\prime}\right)\right|_{X_{0}} \bmod \operatorname{Im} \delta_{0} .
\end{aligned}
$$

\section{The study of obstruction classes}

As explained in the introduction, our goal is to show that the rank of the obstruction map

$$
K_{r-p, 0}\left(X_{0}, L_{0} ; \omega_{X_{0}}\right) \stackrel{\left.D\left(\delta_{t}\right)\right|_{t=0}}{\longrightarrow} K_{r-p-1,1}\left(X_{0}, L_{0} ; \omega_{X_{0}}\right)
$$

is as big as it could be, as this would imply $K_{r-p, 0}\left(X_{t}, L_{t} ; K_{X_{t}}\right)$ is as small as it could be for $t \neq 0$.

Again let us analyze the simpler case $p=r-1$ first. By [Arbarello and Sernesi 1978], the multiplication map

$$
H^{0}\left(X_{t}, L_{t}\right) \otimes H^{0}\left(X_{t}, \omega_{X_{t}}\right) \stackrel{\mu_{t}}{\longrightarrow} H^{0}\left(X_{t}, \omega_{X_{t}} \otimes L_{t}\right)
$$

is already surjective for the general fiber, which implies $K_{1,0}\left(X_{t}, L_{t} ; K_{X_{t}}\right)$ is of the expected dimension. So, we are not proving anything new here, but it is helpful to redo this case via infinitesimal methods, because this method has the potential to generalize.

First notice that

$$
H^{0}\left(X_{0}, L_{0}\right) \otimes H^{0}\left(X_{0}, \omega_{X_{0}}\right) \stackrel{\mu_{0}}{\longrightarrow} H^{0}\left(X_{0}, \omega_{X_{0}} \otimes L_{0}\right)
$$


is not surjective. The problem is that any section in $H^{0}\left(X_{0}, \omega_{X_{0}}\right)$ vanishes at $u$, but there is a section in $H^{0}\left(X_{0}, \omega_{X_{0}} \otimes L_{0}\right)$ not vanishing at $u$. Moreover, $\mu_{0}$ is exactly of corank one. This is because on the $E$ component, $\mu_{0}$ becomes

$$
H^{0}\left(\mathcal{O}_{E}(v)\right) \otimes H^{0}\left(\mathcal{O}_{E}(u)\right) \longrightarrow H^{0}\left(\mathcal{O}_{E}(u+v)\right),
$$

which is of corank 1 (see Figure 1). Since by [Arbarello and Sernesi 1978] (or by the induction hypothesis if one wants an independent proof), the map

$$
H^{0}\left(C, L^{\prime}\right) \otimes H^{0}\left(K_{C}\right) \longrightarrow H^{0}\left(L^{\prime} \otimes K_{C}\right)
$$

is surjective, we see that if a section $\tau \in H^{0}\left(X_{0}, \omega_{X_{0}} \otimes L_{0}\right)$ vanishes at $u$, then it is in the image of $\mu_{0}$. Therefore $k_{1,0}\left(X_{t}, L_{t} ; K_{X_{t}}\right)$ jumps up by one at $t=0$.

Now by the computation of the obstruction class in (4-4),

$$
\left.\left(\tilde{\sigma}^{\prime} \tilde{\omega}^{\prime}\right)\right|_{X_{0}}
$$

is in the image of

$$
H^{0}\left(X_{0}, M\right) \otimes H^{0}\left(X_{0}, N\right) \longrightarrow H^{0}\left(X_{0}, \omega_{X_{0}} \otimes L_{0}\right) .
$$

Since there are always sections in $H^{0}\left(X_{0}, M\right)$ and $H^{0}\left(X_{0}, N\right)$ not vanishing at $u$, we can easily choose $\tilde{\sigma}^{\prime}$ and $\tilde{\omega}^{\prime}$ such that $\left.\left(\tilde{\sigma}^{\prime} \tilde{\omega}^{\prime}\right)\right|_{X_{0}}$ does not vanish at $u$. (Notice that any (global) sections of $M$ and $N$ will extend to nearby fibers.) Therefore there is at least a one-dimensional subspace of $K_{1,0}\left(X_{0}, L_{0} ; K_{X_{0}}\right)$ that does not deform to a nearby fiber, namely $\left(\tilde{\sigma}^{\prime} s_{E}\right)\left(\tilde{\omega}^{\prime} s_{C}\right)$. This means $K_{1,0}\left(X_{t}, L_{t} ; K_{X_{t}}\right)$ is of the expected dimension for $t \neq 0$. This proves the simpler case.

The case for general $p$ is much more delicate. There are two possible ways to show the obstruction classes in (4-7) are not in the image of $\delta_{0}$.

The easier way is to mimic the simpler case is to show $\left.\left(\tilde{\sigma}_{i_{k}}^{\prime} \tilde{\omega}^{\prime}\right)\right|_{X_{0}}$ does not lie in the image of

$$
H^{0}\left(X_{0}, L_{0}\right) \otimes H^{0}\left(X_{0}, \omega_{X_{0}}\right) \stackrel{\mu_{0}}{\longrightarrow} H^{0}\left(X_{0}, \omega_{X_{0}} \otimes L_{0}\right) .
$$

(As we have seen before, $\mu_{0}$ is of corank one.) This will be the case if $\left.\left(\tilde{\sigma}_{i_{k}}^{\prime} \tilde{\omega}^{\prime}\right)\right|_{X_{0}}$ does not vanish at $u$. Then the obstruction class in (4-7) has no chance to be in $\operatorname{Im}\left(\delta_{0}\right)$.

To make this idea more precise, choose a basis $\left\{\sigma_{1}, \ldots, \sigma_{r}\right\}$ of $V$ adapted to $u$, that is, $\left.\sigma_{k}\right|_{C}$ vanishes to order exactly $k$ along $u$ (therefore $\left.\sigma_{k}\right|_{E}=0$ for $k \geq 1$ ). Using the same notation as (4-2) and (4-3), we have that

$$
\left.\left(\tilde{\sigma}_{1}^{\prime} \tilde{\omega}^{\prime}\right)\right|_{X_{0}}
$$

is not in the image of $\mu_{0}$ because $\left.\sigma_{1}\right|_{C}$ vanishes to order exactly 1 at $u$, and any extension $\tilde{\sigma}_{1}=\tilde{\sigma}_{1}^{\prime} \cdot s_{E}$ we choose would have $\tilde{\sigma}_{1}^{\prime}$ nonvanishing at $u$ (because $\left.s_{E}\right|_{C}$ vanishes to order 1 at $u, \tilde{\sigma}_{1}^{\prime}$ does not vanish). Similarly the extension $\tilde{\omega}^{\prime}$ does not 
vanish at $u$. (Although the choice of extensions is not unique, different choices give the same obstruction class modulo $\operatorname{Im}\left(\delta_{0}\right)$.)

However, for $k \geq 2$, because $\left.\sigma_{k}\right|_{C}$ vanishes to order at least 2 at $u$, we could choose a suitable extension $\tilde{\sigma}_{k}$ (modulo $\operatorname{Im}\left(\delta_{0}\right)$ this does not depend on the choice of extension) such that $\tilde{\sigma}_{k}=\tilde{\sigma}_{k}^{\prime \prime} s_{E}^{2}$, and therefore

$$
\left.\left(\tilde{\sigma}_{k}^{\prime} \tilde{\omega}^{\prime}\right)\right|_{E}=\left.\left(\tilde{\sigma}_{k}^{\prime \prime} s_{E} \tilde{\omega}^{\prime}\right)\right|_{E}=0 .
$$

Thus for any $1=i_{1}<i_{2}<i_{3}<\cdots<i_{r-p} \leq r$,

$$
\begin{aligned}
& \left.D\left(\delta_{t}\right)\right|_{t=0}\left(\sigma_{1} \wedge \sigma_{i_{2}} \wedge \cdots \wedge \sigma_{i_{r-p}} \otimes \omega\right) \\
& \quad=-\left.\sigma_{i_{2}} \wedge \cdots \wedge \sigma_{i_{r-p}} \otimes\left(\tilde{\sigma}_{1}^{\prime} \tilde{\omega}^{\prime}\right)\right|_{X_{0}}+\left.\sum_{k=2}^{r-p}(-1)^{k} \sigma_{i_{1}} \wedge \cdots \wedge \hat{\sigma}_{i_{k}} \wedge \cdots \wedge \sigma_{i_{r-p}} \otimes\left(\tilde{\sigma}_{i_{k}}^{\prime} \tilde{\omega}^{\prime}\right)\right|_{X_{0}} .
\end{aligned}
$$

By looking at its restriction to $E$, we see immediately that the set

$$
\left\{\left.D\left(\delta_{t}\right)\right|_{t=0}\left(\sigma_{\mathbf{1}} \wedge \sigma_{i_{2}} \wedge \cdots \wedge \sigma_{i_{r-p}} \otimes \omega\right) \mid 2 \leq i_{2}<i_{3}<\cdots<i_{r-p} \leq r\right\}
$$

is linearly independent in $K_{r-p-1,1}\left(X_{0}, L_{0} ; \omega_{X_{0}}\right)$.

Thus at this point the rank of $\left.D\left(\delta_{t}\right)\right|_{t=0}$ is at least

$$
\left(\begin{array}{c}
r-1 \\
p
\end{array}\right)
$$

and therefore

$$
k_{r-p, 0}\left(X_{t}, L_{t} ; \omega_{X_{t}}\right) \leq\left(\begin{array}{c}
r \\
p
\end{array}\right)-\left(\begin{array}{c}
r-1 \\
p
\end{array}\right)=\left(\begin{array}{c}
r-1 \\
p-1
\end{array}\right)
$$

for $t \neq 0$.

The second way to show obstructions are nontrivial is more delicate. As we have already seen, for $2 \leq i_{1}<\cdots<i_{r-p} \leq r$, restricting to $E$ does not give any information about $\left.D\left(\delta_{t}\right)\right|_{t=0}\left(\sigma_{i_{1}} \wedge \cdots \wedge \sigma_{i_{r-p}} \otimes \omega\right)$, since they all restrict to zero on $E$. We will have to study the restriction of $\left.D\left(\delta_{t}\right)\right|_{t=0}\left(\sigma_{i_{1}} \wedge \cdots \wedge \sigma_{i_{r-p}} \otimes \omega\right)$ to $C$.

Equation (4-7) restricted to $C$ becomes

$$
\begin{aligned}
\left.D\left(\delta_{t}\right)\right|_{t=0}\left(\sigma_{i_{1}} \wedge \cdots\right. & \left.\wedge \sigma_{i_{r-p}} \otimes \omega\right)\left.\right|_{C} \\
& =\left.\sum_{k=1}^{r-p}(-1)^{k} \sigma_{i_{1}} \wedge \cdots \wedge \hat{\sigma}_{i_{k}} \wedge \cdots \wedge \sigma_{i_{r-p}} \otimes\left(\tilde{\sigma}_{i_{k}}^{\prime} \tilde{\omega}^{\prime}\right)\right|_{C} \bmod \operatorname{Im} \delta_{0}
\end{aligned}
$$

Here $\left.\tilde{\sigma}_{i_{k}}^{\prime}\right|_{C} \in H^{0}\left(C, L^{\prime}(-u)\right)$ and is equal to $\sigma_{i_{k}}$ for $i_{k} \geq 1$, if we abuse notation by thinking of $\sigma_{i_{k}}$ as sections of $L^{\prime}(-u)$ instead of $L^{\prime}$. (Thus $\sigma_{1}$ is a section of $L^{\prime}(-u)$ which does not vanish at $u$ and $\sigma_{2}$ vanishes to order 1 at $u$, etc.) On the other hand, $\left.\tilde{\omega}^{\prime}\right|_{C} \in H^{0}\left(K_{C}(2 u)\right)$ and does not vanish at $u$; denote it by $\omega^{\prime}$. With the notation 
above, the obstruction class becomes

$$
\sum_{k=1}^{r-p}(-1)^{k} \sigma_{i_{1}} \wedge \cdots \wedge \hat{\sigma}_{i_{k}} \wedge \cdots \wedge \sigma_{i_{r-p}} \otimes\left(\sigma_{i_{k}} \omega^{\prime}\right)=\delta_{0}\left(\sigma_{i_{1}} \wedge \cdots \wedge \sigma_{i_{r-p}} \otimes \omega^{\prime}\right)
$$

Remark. Here we are still using $\delta_{0}$ to denote the restriction to $C$ of the original Koszul differential $\delta_{0}$ on $X_{0}$. Equation (5-2) does not mean

$$
\left.\left.D\left(\delta_{t}\right)\right|_{t=0}\left(\sigma_{i_{1}} \wedge \cdots \wedge \sigma_{i_{r-p}} \otimes \omega\right)\right|_{C} \in \operatorname{Im}\left(\delta_{0}\right),
$$

since $\omega^{\prime} \notin H^{0}\left(K_{C}\right)$.

Now the nontriviality of obstruction classes on $X_{0}$ boils down to a question on $\left(C^{\prime}, L^{\prime}\right)$ :

Theorem 5.1. Let $C$ be a general curve of genus $g, L^{\prime}$ a $g_{d}^{r}$ on $C$ such that $K_{r-p, 0}\left(C, L^{\prime} ; K_{C}\right)=0$ and $\left\{\sigma_{0}, \ldots, \sigma_{r}\right\}$ is a basis of $H^{0}\left(C, L^{\prime}\right)$ adapted to a general point $u \in C$, and let $\omega^{\prime} \in H^{0}\left(C, K_{C}(2 u)\right) \backslash H^{0}\left(C, K_{C}\right)$. Consider the obstruction classes

$$
\left\{\delta_{0}\left(\sigma_{i_{1}} \wedge \cdots \wedge \sigma_{i_{r-p}} \otimes \omega^{\prime}\right) \mid 2 \leq i_{1}<\cdots<i_{r-p} \leq r\right\} \subset K_{r-p-1,1}\left(C, L^{\prime} ; K_{C}\right) .
$$

(a) If these classes are linearly independent in $K_{r-p-1,1}\left(C, L^{\prime}, K_{C}\right)$, then

$$
K_{r-p, 0}\left(X_{t}, L_{t} ; \omega_{X_{t}}\right) \cong K_{p-1,2}\left(X_{t}, L_{t}\right)^{\vee}=0 .
$$

(b) On the other hand, if these classes span $K_{r-p-1,1}\left(C, L^{\prime} ; K_{C}\right)$, then

$$
k_{r-p, 0}\left(X_{t}, L_{t} ; \omega_{X_{t}}\right) \leq\left(\begin{array}{c}
r-1 \\
p-1
\end{array}\right)-k_{r-p-1,1}\left(C, L^{\prime} ; K_{C}\right)=-b_{p+1}\left(X_{t}, L_{t}\right),
$$

which implies $K_{p, 1}\left(X_{t}, L_{t}\right)=0$.

Proof. The hypothesis in case (a) implies that $\left.D\left(\delta_{t}\right)\right|_{t=0}$ in (4-1) is either injective, which means no elements in $K_{r-p, 0}\left(X_{0}, L_{0} ; \omega_{X_{0}}\right)$ will extend to nearby. In case (b), the rank of $\left.D\left(\delta_{t}\right)\right|_{t=0}$ is

$$
k_{r-p-1,1}\left(C, L^{\prime} ; K_{C}\right)+\left(\begin{array}{c}
r-1 \\
p
\end{array}\right)
$$

which implies that

$$
k_{r-p, 0}\left(X_{t}, L_{t} ; \omega_{X_{t}}\right) \leq\left(\begin{array}{c}
r-1 \\
p-1
\end{array}\right)-k_{r-p-1,1}\left(C, L^{\prime} ; K_{C}\right)=-b_{p+1}\left(X_{t}, L_{t}\right) .
$$

Therefore only a subspace of $K_{r-p, 0}\left(X_{0}, L_{0} ; \omega_{X_{0}}\right)$ of correct dimension will extend to nearby fibers to first order. 
Now we give a sufficient condition for the obstruction classes in (5-3) to be linearly independent. Consider the diagram of complexes

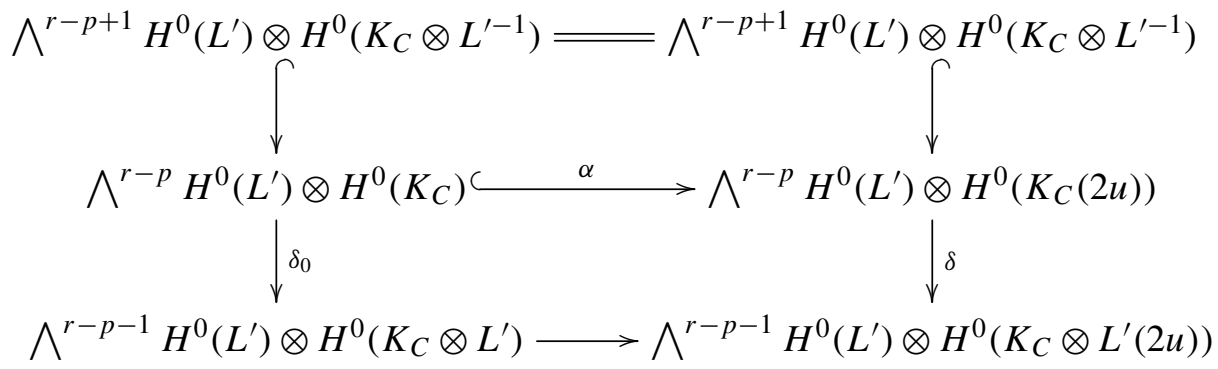

Lemma 5.2. Under the same assumptions as Theorem 5.1, if the right column of the above diagram is exact in the middle (the left column is exact by assumption), then the obstruction classes in (5-3) are linearly independent modulo $\operatorname{Im}\left(\delta_{0}\right)$. As a consequence of Theorem 5.1 then, $K_{r-p, 0}\left(X_{t}, L_{t} ; \omega_{X_{t}}\right)=0$.

Proof. The assumption implies that

$$
\operatorname{Ker}(\delta)=\operatorname{Ker}\left(\delta_{0}\right) \cong \bigwedge^{r-p+1} H^{0}\left(L^{\prime}\right) \otimes H^{0}\left(K_{C} \otimes L^{\prime-1}\right) .
$$

If a linear combination of the obstruction classes $\delta_{0}\left(\sigma_{i_{1}} \wedge \cdots \wedge \sigma_{i_{r-p}} \otimes \omega^{\prime}\right)$ is equal to $\delta_{0}(c)$ for some $c \in \bigwedge^{r-p} H^{0}\left(L^{\prime}\right) \otimes H^{0}\left(K_{C}\right)$, then the same linear combination of the $\left\{\sigma_{i_{1}} \wedge \cdots \wedge \sigma_{i_{r-p}} \otimes \omega^{\prime}\right\}$ minus $\alpha(c)$ is in $\operatorname{Ker}(\delta)=\operatorname{Ker}\left(\delta_{0}\right)$. This contradicts the fact that

$$
\left\{\sigma_{i_{1}} \wedge \cdots \wedge \sigma_{i_{r-p}} \otimes \omega^{\prime}\right\}
$$

is linearly independent in $\bigwedge^{r-p} H^{0}\left(L^{\prime}\right) \otimes H^{0}\left(K_{C}(2 u)\right)$ modulo the image of $\alpha$.

To end this section, we give a proof of Theorem 1.3.

Proof of Theorem 1.3. There are two cases:

(a) $K_{p, 1}\left(C, L^{\prime}\right)=0$ : By Proposition $3.1, K_{p, 1}\left(X_{0}, L_{0}\right)=0$, and $\mathrm{GV}(p)_{g+1, d+1}^{r}$ follows from upper-semicontinuity of Koszul cohomology.

(b) $K_{p-1,2}\left(C, L^{\prime}\right) \cong K_{r-p, 0}\left(C, L, ; K_{C}\right)^{\vee}=0$ : Starting from the defining sequence for the kernel bundle $M_{L^{\prime}}$,

$$
0 \longrightarrow M_{L^{\prime}} \longrightarrow H^{0}\left(L^{\prime}\right) \otimes \mathcal{O}_{C} \longrightarrow L^{\prime} \longrightarrow 0,
$$

taking the $(r-p)$-th wedge, twisting by $K_{C}$ (respectively $\left.K_{C}(2 u)\right)$ and then taking global sections, we get

$0 \longrightarrow \bigwedge^{r-p} M_{L^{\prime}} \otimes K_{C} \longrightarrow \bigwedge^{r-p} H^{0}\left(L^{\prime}\right) \otimes H^{0}\left(K_{C}\right)$

$$
\stackrel{\delta_{0}}{\longrightarrow} \bigwedge^{r-p-1} H^{0}\left(L^{\prime}\right) \otimes H^{0}\left(K_{C} \otimes L^{\prime}\right) \longrightarrow 0,
$$


and therefore

$$
\operatorname{Ker}\left(\delta_{0}\right)=H^{0}\left(C, \bigwedge^{r-p} M_{L^{\prime}} \otimes K_{C}\right)
$$

Similarly,

$$
\operatorname{Ker}(\delta)=H^{0}\left(C, \wedge^{r-p} M_{L^{\prime}} \otimes K_{C}(2 u)\right)
$$

If

$$
h^{0}\left(C, \bigwedge^{r-p} M_{L^{\prime}} \otimes K_{C}\right)=h^{0}\left(C, \bigwedge^{r-p} M_{L^{\prime}} \otimes K_{C}(2 u)\right),
$$

we conclude that

$$
\operatorname{Ker}\left(\delta_{0}\right) \cong \operatorname{Ker}(\delta),
$$

which implies $K_{r-p, 0}\left(X_{t}, L_{t} ; \omega_{X_{t}}\right) \cong K_{p, 2}\left(X_{t}, L_{t}\right)^{\vee}=0$ by Lemma 5.2.

\section{Some applications to the maximal rank conjecture}

In the case $p=1$, we can reduce condition (1-4) in Theorem 1.3 to a statement about the tangential variety $T C$ of $C$; namely, the existence of a quadric containing $C$ but not containing $T C$. The condition on the tangential variety is quite interesting in its own right. Theorem 1.4 follows immediately from Theorem 1.3 and Lemma 6.1.

Lemma 6.1. For a general $L^{\prime}=g_{d}^{r}$ on a general curve $C$ of genus $g$ such that $K_{0,2}\left(C, L^{\prime}\right)=0$ (i.e., $\mu$ in (1-1) is surjective), if there exists a quadric $Q \subset \mathbb{P}^{r}$ containing $\phi_{\left|L^{\prime}\right|}(C)$ but not containing its tangential surface $T C:=\bigcup_{u \in C} T_{u} C \subset \mathbb{P}^{r}$, then

$$
H^{0}\left(C, \wedge^{r-1} M_{L^{\prime}} \otimes K_{C}\right)=H^{0}\left(C, \wedge^{r-1} M_{L^{\prime}} \otimes K_{C}(2 u)\right) .
$$

Proof. Notice that

$$
\bigwedge^{r} M_{L^{\prime}} \cong L^{\prime-1}
$$

and therefore

$$
\bigwedge^{r-1} M_{L^{\prime}}^{\vee} \cong M_{L^{\prime}} \otimes L^{\prime}
$$

By the Riemann-Roch theorem, it suffices to show that

$$
h^{0}\left(M_{L^{\prime}} \otimes L^{\prime}(-2 u)\right)=h^{0}\left(M_{L^{\prime}} \otimes L^{\prime}\right)-2 r .
$$

The $\geq$ part is automatically true, and only the $\leq$ part needs to be proved. 
We have a diagram with exact columns

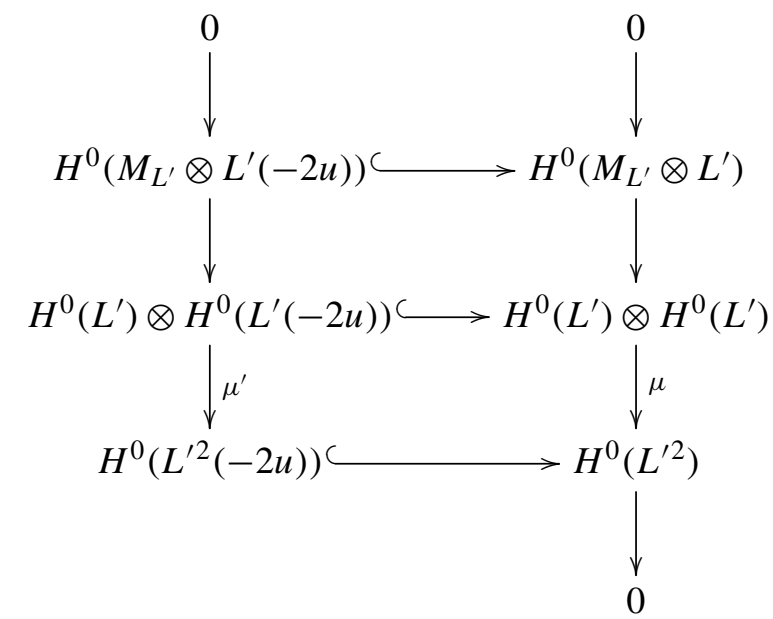

We need to show

$$
\operatorname{dim}_{\mathbb{C}} \operatorname{Ker}\left(\mu^{\prime}\right) \leq \operatorname{dim}_{\mathbb{C}} \operatorname{Ker}(\mu)-2 r .
$$

Let $H_{u}:=H^{0}\left(L^{\prime}\right) \otimes H^{0}\left(L^{\prime}(-2 u)\right)$, and $\bar{H}_{u}$ its image in

$$
\frac{H^{0}\left(L^{\prime}\right) \otimes H^{0}\left(L^{\prime}\right)}{\bigwedge^{2} H^{0}\left(L^{\prime}\right)} \cong S^{2} H^{0}\left(L^{\prime}\right)
$$

$\bar{H}_{u}$ is the space of quadrics which contain the tangent line of $C$ at $u$.

We have

$$
\operatorname{Ker}\left(\mu^{\prime}\right)=\operatorname{Ker}(\mu) \cap H_{u} .
$$

By hypothesis, $\overline{\operatorname{Ker}(\mu)} \nsubseteq \bar{H}_{u}$ for general $u$ (since $Q \notin \bar{H}_{u}$ ), and it follows that

$$
\begin{aligned}
\operatorname{dim}_{\mathbb{C}}\left(\overline{\operatorname{Ker}\left(\mu^{\prime}\right)}\right)=\operatorname{dim}_{\mathbb{C}}\left(\overline{\operatorname{Ker}(\mu) \cap H_{u}}\right) & \leq \operatorname{dim}_{\mathbb{C}}\left(\overline{\operatorname{Ker}(\mu)} \cap \bar{H}_{u}\right) \\
& \leq \operatorname{dim}_{\mathbb{C}}(\overline{\operatorname{Ker}(\mu)})-1=: m-1 .
\end{aligned}
$$

Thus

$$
\begin{aligned}
\operatorname{dim}_{\mathbb{C}}\left(\operatorname{Ker}\left(\mu^{\prime}\right)\right) & \leq m-1+\operatorname{dim}_{\mathbb{C}}\left(\bigwedge^{2} H^{0}\left(L^{\prime}\right) \cap H_{u}\right) \\
& =m-1+\operatorname{dim}_{\mathbb{C}}\left(\bigwedge^{2} H^{0}\left(L^{\prime}(-2 u)\right)\right) \\
& =m-1+\left(\begin{array}{c}
r-1 \\
2
\end{array}\right)=m+\left(\begin{array}{c}
r+1 \\
2
\end{array}\right)-2 r \\
& =\operatorname{dim}_{\mathbb{C}}(\operatorname{Ker}(\mu))-2 r .
\end{aligned}
$$

Let us go the proof of Corollary 1.5. The numerical assumption in Corollary 1.5 turns out to be a technical assumption needed to verify the assumption about $T C$ in Theorem 1.4(b). This is equivalent to the numerical assumption in Lemma 6.2. By 
the Appendix, if $L^{\prime}$ is a general nonspecial $g_{2 r-3}^{r}$ on a general curve $C$ of genus $r-3$, the number of quadrics containing $T C$ is at most

$$
\left(\begin{array}{c}
r-4 \\
2
\end{array}\right) \text {. }
$$

Lemma 6.2. Let $C \subset \mathbb{P}^{r}$ be a general curve of genus $g$ embedded by $L^{\prime}$ a general $g_{d}^{r}$ with $h^{1}\left(L^{\prime}\right) \leq 1$. Suppose

$$
\left(\begin{array}{c}
r+2 \\
2
\end{array}\right)-(2 d-g+1)>\left(\begin{array}{c}
r-4 \\
2
\end{array}\right)
$$

(i.e., the number of independent quadrics containing $C$ is at least $\left.\left(\begin{array}{c}r-4 \\ 2\end{array}\right)\right)$; then there exists a quadric $Q$ on $\mathbb{P}^{r}$ containing $C$ but not containing $T C$.

Proof. Degenerate $\left(C, L^{\prime}\right)$ to $\left(C_{0}, L_{0}^{\prime}\right)$, where $C_{0}$ is a nodal curve with two smooth components $Y$ and $Z$ meeting at a general point $u$. Depending on the value of $h^{1}\left(L^{\prime}\right)$, there are two cases:

(a) $h^{1}\left(L^{\prime}\right)=0$ : In this case, $L^{\prime}=g_{g+r}^{r}$ for $g \geq 0$. If $0 \leq g \leq r-3$, take $g_{Y}=0$, $g_{Z}=g,\left.L_{0}^{\prime}\right|_{Y}=\mathcal{O}_{\mathbb{P}^{1}}(r)$ and $\left.L_{0}^{\prime}\right|_{Z}=g_{g}^{0}$. (One could easily show such a $\left(C_{0}, L_{0}^{\prime}\right)$ can deform to $\left(C, L^{\prime}\right)$.) Since there are only

$$
\left(\begin{array}{c}
r-2 \\
2
\end{array}\right)
$$

quadrics containing the tangential variety of the rational normal curve in $\mathbb{P}^{r}$ (see the Appendix) and in this range of $g$, the number of quadrics containing $C$ is at least

$$
\left(\begin{array}{c}
r+2 \\
2
\end{array}\right)-(2 d-g+1)=\left(\begin{array}{c}
r+2 \\
2
\end{array}\right)-(g+2 r+1)>\left(\begin{array}{c}
r-2 \\
2
\end{array}\right),
$$

we conclude that there exists a quadric containing the nearby fiber $C$ but not containing $T C$.

If $g>r-3$, we take $g_{Y}=r-3, g_{Z}=g-r+3,\left.L_{0}^{\prime}\right|_{Y}=g_{2 r-3}^{r}$ (a general one) and $\left.L_{0}^{\prime}\right|_{Z}=g_{g-r+3}^{0}$. By Proposition A.1 in the Appendix, the number of quadrics containing $T C$ for nearby $C$ is at most $\left(\begin{array}{c}r-4 \\ 2\end{array}\right)$. By the numerical hypothesis, we get our conclusion.

(2) $h^{1}\left(L^{\prime}\right)=1$ : The argument is similar to the above, except that we need to deal with $L^{\prime}=g_{g+r-1}^{r}$ for $g \geq r+1$. Again, if $r+1 \leq g \leq 2 r-2$, we take $g_{Y}=0$, $Z=C,\left.L_{0}^{\prime}\right|_{Y}=\mathcal{O}_{\mathbb{P}^{1}}(r)$ and $\left.L_{0}^{\prime}\right|_{Z}=L^{\prime}(-r u)=g_{g-1}^{0}$.

If $g>2 r-2$, take $g_{Y}=r-3, g_{Z}=g-r+3,\left.L_{0}^{\prime}\right|_{Y}=g_{2 r-3}^{r},\left.L_{0}^{\prime}\right|_{Z}=g_{g-r+2}^{0}$. Here $\left.L_{0}^{\prime}\right|_{Z}$ comes from a general $g_{g+2}^{r}$ on $Z$ twisted by $\mathcal{O}_{Z}(-r u)$. The rest of the argument is exactly the same as in case (a). 
Proof of Corollary 1.5. First notice that, by Corollary 2.2, to show projective normality of a general pair, it suffices to show (1-1) is surjective. We will fix $h^{1}$ and $r$ and induct on $g$.

For the $h^{1}=0$ case, we start with the fact that a rational normal curve is projectively normal (i.e., (MRC) $)_{0, r}^{r}$ holds). For the $h^{1}=1$ case, we use the fact that a general canonical curve is projectively normal (i.e., (MRC) ${ }_{r+1,2 r}^{r}$ holds). Now assuming (MRC) ${ }_{g, d}^{r}$ holds, by Lemma 6.2, as long as

$$
\left(\begin{array}{c}
r+2 \\
2
\end{array}\right)-(2 d-g+1)>\left(\begin{array}{c}
r-4 \\
2
\end{array}\right)
$$

Theorem 1.4(b) is satisfied, which implies (MRC) ${ }_{g+1, d+1}^{r}$ (which is equivalent to projective normality). Plugging $d=g+r-h^{1}$ into (6-1), we immediately get the bound on $d$ as in the statement of the theorem.

Proof of Corollary 1.8. The $r=1,2$ cases is trivial. The arguments for $r=3,4$ are completely similar, so we will only prove the case $r=4$. Again, we induct on $g$. First suppose we have proved (MRC) for the base cases $g=5 h^{1}, L=g_{4 h^{1}+4}^{4}$. Then notice that for $r=4,\left(\begin{array}{c}r-4 \\ 2\end{array}\right)=0$, and therefore there is no quadric containing the tangential variety in Theorem 1.4(b). Thus (MRC) $)_{g, d}^{4}$ implies (MRC) $)_{g+1, d+1}^{4}$. It remains to prove (MRC) for the base cases. When $h^{1} \leq 1$, (MRC) ${ }_{5 h^{1}, 4 h^{1}+4}^{4}$ is clear. If $h^{1} \geq 2$, we need to show $\mu$ in (1-1) is injective. For $h^{1}=2$, (MRC) ${ }_{10,12}^{4}$ is well known and is proved in [Farkas and Popa 2005]. If $h^{1} \geq 3$, we could degenerate again to $C_{0}=Y \cup Z$ with $g_{Y}=10, g_{X}=5 h^{1}-10,\left.L_{0}\right|_{Y}=g_{12}^{4}$, $\left.L_{0}\right|_{Z}=g_{4 h^{1}-8}^{0}=g_{4 h^{1}-4}^{4}(-4 u)$. Again it is easy to check such $\left(C_{0}, L_{0}\right)$ is smoothable in $\mathcal{G}_{5 h^{1}, 4 h^{1}+4}^{4}$ (see [Wang 2013, Corollary 6.1] for details). The injectivity of $\mu$ in this case follows from the same argument as in Proposition 3.1.

It was also proved in [Farkas 2009] that for any integer $s \geq 1,(\mathrm{MRC})_{s(2 s+1), 2 s(s+1)}^{2 s}$ holds. In this case, $\rho=0$ and $h^{1}=s$. Thus by Theorem 1.4 (a), we have the following corollary:

Corollary 6.3. (MRC) $)_{s(2 s+1)+k, 2 s(s+1)+k}^{2 s}$ holds for all $s \geq 1, k \geq 0$; that is, (MRC) holds if $r=2 h^{1}$.

\section{Higher syzygies}

As we mentioned in the introduction, the difficulty in generalizing the inductive argument to higher syzygies is due to the lack of known cases to start the induction with and the lack of an analog of Theorem 1.4 for higher syzygies. Nevertheless, we collect some vanishing results we could obtain in this section.

Proposition 7.1. For $L$ a general $g_{d}^{r}$ on a general curve $X$ with $g \geq r+1$, $K_{p, 1}(X, L)=0$ for $p \geq\lfloor(r+1) / 2\rfloor$. 
Proof. Start with the case $g_{X}=r+1, L^{\prime}=K_{X}$. Thanks to Voisin's solution to the generic Green conjecture, $K_{p, 1}\left(X, K_{X}\right)=0$ for $p \geq\lfloor(r+1) / 2\rfloor$. If $g>r+1$, we degenerate to $X_{0}=Y \cup Z$ with $g_{Y}=r+1, g_{Z}=g-r-1,\left.L_{0}\right|_{Y}=K_{Y}$, $\left.L_{0}\right|_{Z}=g_{d-2 r}^{0}=g_{d-r}^{r}(-r u)$. The statement then follows from the same argument as in Proposition 3.1.

Remark. Using the same degeneration as in Proposition 7.1, we also have

$$
k_{p, 1}(X, L) \leq k_{p .1}\left(Y, K_{Y}\right)=\left[\left(\begin{array}{c}
r-1 \\
p
\end{array}\right)-\left(\begin{array}{c}
r-1 \\
p-1
\end{array}\right)\right] r+\left(\begin{array}{c}
r+1 \\
p
\end{array}\right)-\left(\begin{array}{c}
r+1 \\
p+1
\end{array}\right)
$$

for $1 \leq p<\lfloor(r+1) / 2\rfloor$. We will improve this bound using an infinitesimal argument.

Even though we do not have an analog of Theorem 1.4, when $g$ is not too big compared to $p$, the inductive argument still go through:

Lemma 7.2. Let $C$ be a general curve of genus $g$ and $L^{\prime}$ a $g_{d}^{r}$ with $h^{1}\left(L^{\prime}\right)=1$. If $p \leq r-\lfloor(g+1) / 2\rfloor$, then the sequence

$$
\begin{aligned}
\bigwedge^{r-p+1} H^{0}\left(L^{\prime}\right) \otimes H^{0}\left(K_{C} \otimes L^{\prime-1}\right) \longleftrightarrow & \bigwedge^{r-p} H^{0}\left(L^{\prime}\right) \otimes H^{0}\left(K_{C}(2 u)\right) \\
& \stackrel{\delta}{\longrightarrow} \bigwedge^{r-p-1} H^{0}\left(L^{\prime}\right) \otimes H^{0}\left(K_{C} \otimes L^{\prime}(2 u)\right)
\end{aligned}
$$

is exact in the middle.

Proof. Let $t \in H^{0}\left(K_{C} \otimes L^{\prime-1}\right)$ be a generator. Multiplication by $t$ gives an embedding

$$
H^{0}\left(L^{\prime}\right) \stackrel{\cdot t}{\longrightarrow} H^{0}\left(K_{C}\right) .
$$

Denote its image by $W$. Let $C^{\prime}$ be the image of $C$ under the map given by $\left|K_{C}(2 u)\right|$. $C^{\prime}$ is of arithmetic genus $g+1$ and has a cusp. We can identify $H^{0}\left(C, K_{C}(2 u)\right)$ with $H^{0}\left(C^{\prime}, \omega_{C^{\prime}}\right)$, where $\omega_{C^{\prime}}$ is the dualizing sheaf of $C^{\prime}$. With the above notation, we can identify the cohomology group in question with $K_{r-p, 1}\left(C^{\prime}, \omega_{C^{\prime}} ; W\right) \subset$ $K_{r-p, 1}\left(C^{\prime}, \omega_{C^{\prime}}\right)$. Since curves in $K 3$ surfaces satisfy the Green conjecture (see [Voisin 2002; 2005]), by degenerating $C^{\prime}$ to a cuspidal curve in $K 3$ surface, we have

$$
K_{r-p, 1}\left(C^{\prime}, \omega_{C^{\prime}}\right)=0
$$

for $r-p \geq\lfloor(g+1) / 2\rfloor$.

Again we start our induction with a general curve of genus $r+1$, and $L^{\prime}=K_{C}$. For $p<\lfloor(r+1) / 2\rfloor$, we have

$$
K_{p-1,2}\left(C, K_{C}\right)^{\vee} \cong K_{r-p, 1}\left(C, K_{C}\right)=0 .
$$

Now we apply the construction in Section 3. By Lemma 5.2 and Lemma 7.2, we get: 
Proposition 7.3. For L a general $g_{d}^{r}$ on a general curve $X$ with $h^{1}(L)=1$ :

(a) $k_{p-1,2}(X, L)=k_{r-p, 0}\left(X, L ; K_{X}\right)=0$ if $p \leq r-\lfloor g / 2\rfloor$.

(b) $k_{p-1,2}(X, L) \leq(g-2 r+2 p-1)\left(\begin{array}{c}r-1 \\ p-1\end{array}\right)$ if $p>r-\lfloor g / 2\rfloor$.

Proof. Again we start our induction on $g$ with a general curve of genus $r+1$ and $L^{\prime}=K_{C}$. We always have

$$
K_{p-1,2}\left(C, K_{C}\right)^{\vee} \cong K_{r-p, 1}\left(C, K_{C}\right)=0
$$

for $r-p \geq\lfloor(r+1) / 2\rfloor$.

Now we apply the construction in Section 3. If $\lfloor g / 2\rfloor \leq r-p$, Lemmas 5.2 and 7.2 apply, and we get (a).

When $\lfloor g / 2\rfloor$ gets past $r-p$ (or equivalently $g>2 r-2 p+1$ ), we nevertheless have estimate (5-1) for each attached elliptic tail. Thus the bound in (b) follows.

Combining the results of Propositions 7.1 and 7.3, we get Theorem 1.7.

Remark. For line bundles with $h^{1}=1$, the assumption $p \leq r-\lfloor g / 2\rfloor$ is equivalent to the condition that $d \geq 2 g-2+p-\lfloor(g-1) / 2\rfloor$. Thus Proposition 7.3 is the generic version of the generalized Green-Lazarsfeld conjecture [1986] for special linear series. However this generic version is known to follow from the generic Green conjecture (see [Aprodu and Farkas 2011, Proposition 4.30]). It seems to the author that the bound in (b) is new.

\section{Appendix}

We prove the following statement, which is needed in the proof of Corollary 1.5.

Proposition A.1. For a general curve $C$ of genus $r-3$ embedded in $\mathbb{P}^{r}$ by a general $g_{2 r-3}^{r}$, the number of quadrics containing TC is at most

$$
\left(\begin{array}{c}
r-4 \\
2
\end{array}\right)
$$

Consider the rational normal curve $C$ of degree $d$ in $\mathbb{P}^{d}$. It is well known that there are

$$
\left(\begin{array}{l}
d \\
2
\end{array}\right)
$$

independent quadrics containing $C$. Denote them by $\Delta_{a, b}$ for $0 \leq a<b \leq d-1$, where $\Delta_{a, b}$ is the $2 \times 2$ minor corresponding to columns $a$ and $b$ of the matrix

$$
\left(\begin{array}{cccccc}
x_{0} & x_{1} & x_{2} & \cdots & x_{d-2} & x_{d-1} \\
x_{1} & x_{2} & x_{3} & \cdots & x_{d-1} & x_{d}
\end{array}\right)
$$

with the usual convention that $\Delta_{a, b}=-\Delta_{b, a}$. 
It is proved in [Eisenbud 1992] that there are

$$
\left(\begin{array}{c}
d-2 \\
2
\end{array}\right)
$$

quadrics containing $T C$. They are

$$
\Gamma_{a, b}=\Delta_{a+2, b}-2 \Delta_{a+1, b+1}+\Delta_{a, b+2}
$$

for $0 \leq a, b \leq d-3$.

Now, consider the projection $C^{\prime}$ of $C$ to $\mathbb{P}^{r}(d=2 r-3, r \geq 3)$ given by

$$
t \longrightarrow\left[1, t^{2}, t^{4}, \ldots, t^{2 r-6}, t^{2 r-5}, t^{2 r-4}, t^{2 r-3}\right] .
$$

$C^{\prime}$ has arithmetic genus $r-3$ and has a unique singular point at $t=0$ locally isomorphic to $\operatorname{Spec}\left(\mathbb{C}\left[t^{2}, t^{2 r-5}\right]\right)$.

Lemma A.2. The complete linear system $\left|\mathcal{O}_{C^{\prime}}(1)\right|$ has projective dimension $r$; that is, $C^{\prime} \subset \mathbb{P}^{r}$ is linearly normal. As a consequence, $C^{\prime}$ is smoothable in $\mathbb{P}^{r}$.

Proof. Let $L_{l}=\operatorname{span}\left\{P_{1}, P_{3}, \ldots, P_{2 k-1}\right\} \subset \mathbb{P}^{2 r-3}$, where

$$
P_{i}=\left[0,0, \ldots, 1^{(i-\text { th })}, \ldots, 0\right], \quad i=0,1, \ldots, r,
$$

and denote by $C_{k} \subset \mathbb{P}^{2 r-3-k}$ the projection of $C$ with center $L_{k}$. The curve $C_{k}$ has a unique singular point locally isomorphic to $\operatorname{Spec}\left(\mathbb{C}\left[t^{2}, t^{2 k+1}\right]\right)$. Note that $C^{\prime}=C_{r-3} \subset \mathbb{P}^{r}$. We use induction to show that the complete linear system $\left.\mathcal{O}_{\mathbb{P}^{2 r-3-k}(1)}\right|_{C_{k}}$ has projective dimension $2 r-3-k$. The natural projection map $P r_{k}: C_{k} \rightarrow C_{k+1}$ induces an inclusion $H^{0}\left(\mathcal{O}_{C_{k+1}}(1)\right) \subset H^{0}\left(\mathcal{O}_{C_{k}}(1)\right)$. By the inductive hypothesis, $h^{0}\left(\left(\mathcal{O}_{C_{k}}(1)\right)=h^{0}\left(\mathcal{O}_{\mathbb{P}^{2 r-3-k}}(1)\right)=2 r-2-k\right.$. Since we obtain $C_{k+1}$ from $C_{k}$ by projection from a point, $h^{0}\left(\left(\mathcal{O}_{C_{k+1}}(1)\right) \geq h^{0}\left(\left(\mathcal{O}_{C_{k}}(1)\right)-1\right.\right.$. Since $C_{k+1}$ has arithmetic genus one higher than $C_{k}, H^{0}\left(\mathcal{O}_{C_{k+1}}(1)\right) \nsubseteq H^{0}\left(\mathcal{O}_{C_{k}}(1)\right)$. Thus $h^{0}\left(\left(\mathcal{O}_{C_{k+1}}(1)\right)=2 r-3-k\right.$. For the last statement, note that the curve $C^{\prime}$ has only a plane curve singularity, and thus is smoothable (as an abstract curve). Moreover, since $h^{0}\left(\mathcal{O}_{C^{\prime}}(1)\right)=r+1, \mathcal{O}_{C^{\prime}}(1)$ is a complete nonspecial $g_{2 r-3}^{r}$. For any oneparameter smoothing $\left(C_{t}, L_{t}\right)$ of the pair $\left(C^{\prime}, \mathcal{O}_{C^{\prime}}(1)\right)$, since $h^{0}$ of the central fiber does not jump up, all $r+1$ global sections of $\mathcal{O}_{C^{\prime}}(1)$ deform to $L_{t}$.

Proof of Proposition A.1. We could explicitly compute the quadrics containing $T C^{\prime}$ : they are just quadrics in $\mathbb{P}^{2 r-3}$ containing $T C$ with singular locus containing the center of projection $L_{r-3}=\operatorname{span}\left\{P_{1}, P_{3}, \ldots, P_{2 r-7}\right\}$. Now if we think of each quadric $\Gamma_{a, b}$ as a $(2 r-2) \times(2 r-2)$ symmetric matrix, we are just looking for matrices $Q \in S_{\Gamma}:=\operatorname{span}\left\{\Gamma_{a, b} \mid 0 \leq a<b \leq 2 r-6\right\}$ such that $L_{r-3} \subset \operatorname{Ker} Q$. (We think of $Q$ as a linear operator on $\mathbb{C}^{2 r-2}$ and $L_{r-3}$ as a subspace of $\mathbb{C}^{2 r-2}$.) 
Notice that each $\Gamma_{a, b}$, as a matrix, can have nonzero entries at the $(i, j)$-spot $(0 \leq i, j \leq 2 r-3)$ only if

$$
i+j=a+b+3 \text {. }
$$

Stated differently, each $\Gamma_{a, b}$, as a matrix, is supported on one of the diagonals.

For each $4 \leq k \leq 4 r-10$, there are $(\lfloor k / 2\rfloor-1)$ of the $\Gamma_{a, b}$ contributing to nonzero entries on the line

$$
i+j=k, \quad \text { for } 4 \leq k \leq 2 r-3,
$$

and $(2 r-4-\lfloor(k+1) / 2\rfloor)$ of the $\Gamma_{a, b}$ if $2 r-3<k \leq 4 r-10$.

Write

$$
S_{\Gamma}=\bigoplus_{k=4}^{4 r-10} S_{k}
$$

where $S_{k}=\operatorname{span}\left\{\Gamma_{a, b} \mid 0 \leq a<b \leq 2 r-6, a+b=k-3\right\}$. It is obvious that if $Q \in S_{\Gamma}$ vanishes on $L_{r-3}$, then its $S_{k}$ component also vanishes on $L_{r-3}$. Thus it suffices to count how many quadrics in each $S_{k}$ vanish on $L_{r-3}$.

Let's just consider the case $4 \leq k \leq 2 r-3$; the other case is similar.

When $k$ is odd, vanishing on $L_{r-3}$ imposes $(k-1) / 2$ independent conditions on $S_{k}$, more than the dimension of $S_{k}$. Thus no quadric in $S_{k}$ vanishes on $L_{r-3}$.

When $k$ is even, vanishing on $L_{r-3}$ only imposes $\lceil k / 4\rceil$ independent conditions. We conclude that for $4 \leq k \leq 2 r-3$, there are

$$
\sum_{\substack{8 \leq k \leq 2 r-4 \\ k \text { even }}}\left(\frac{k}{2}-1-\left\lceil\frac{k}{4}\right\rceil\right)=\left\lfloor\frac{r^{2}-8 r+16}{4}\right\rfloor
$$

quadrics containing $T C^{\prime}$ (if $r \leq 5$ there are none!).

Similarly, for $2 r-3<k \leq 4 r-10$, we count that there are

$$
\left\lfloor\frac{r^{2}-8 r+16}{4}\right\rfloor-\left\lfloor\frac{r-4}{2}\right\rfloor
$$

quadrics containing $T C^{\prime}$.

So we get a total of

$$
\left\lfloor\frac{r^{2}-8 r+16}{4}\right\rfloor+\left\lfloor\frac{r^{2}-8 r+16}{4}\right\rfloor-\left\lfloor\frac{r-4}{2}\right\rfloor=\left(\begin{array}{c}
r-4 \\
2
\end{array}\right)
$$

quadrics containing $T C^{\prime}$. By specializing to $C^{\prime}$, we conclude our proof. 


\section{Acknowledgements}

This work is a continuation of my thesis project. I would like to thank my thesis adviser Herb Clemens for suggesting the problem and method, and his constant support for this work. I would also like to thank Aaron Bertram, Gavril Farkas and Joe Harris for generously sharing their ideas on this problem. Last but not least, I thank the referee for the helpful comments and suggestions to improve the paper.

\section{References}

[Aprodu 2004] M. Aprodu, "Green-Lazarsfeld gonality conjecture for a generic curve of odd genus", Int. Math. Res. Not. 2004:63 (2004), 3409-3416. MR 2005k:14012 Zbl 1072.14036

[Aprodu 2005] M. Aprodu, "Remarks on syzygies of $d$-gonal curves", Math. Res. Lett. 12:2-3 (2005), 387-400. MR 2006d:14028 Zbl 1084.14032

[Aprodu and Farkas 2011] M. Aprodu and G. Farkas, "Green's conjecture for curves on arbitrary K3 surfaces”, Compos. Math. 147:3 (2011), 839-851. MR 2012e:14006 Zbl 1221.14039

[Aprodu and Nagel 2010] M. Aprodu and J. Nagel, Koszul cohomology and algebraic geometry, University Lecture Series 52, American Mathematical Society, Providence, RI, 2010. MR 2011f:14051 Zbl 1189.14001

[Arbarello and Cornalba 1981] E. Arbarello and M. Cornalba, "Su una congettura di Petri", Comment. Math. Helv. 56:1 (1981), 1-38. MR 82k:14029 Zbl 0505.14002

[Arbarello and Sernesi 1978] E. Arbarello and E. Sernesi, "Petri's approach to the study of the ideal associated to a special divisor", Invent. Math. 49:2 (1978), 99-119. MR 80c:14020 Zbl 0399.14019

[Ballico 1996] E. Ballico, "On the minimal free resolution of general embeddings of curves", Pacific J. Math. 172:2 (1996), 315-319. MR 97d:14042 Zbl 0848.14007

[Ballico and Fontanari 2010a] E. Ballico and C. Fontanari, "Normally generated line bundles on general curves", J. Pure Appl. Algebra 214:6 (2010), 837-840. MR 2011b:14013 Zbl 1184.14039

[Ballico and Fontanari 2010b] E. Ballico and C. Fontanari, "Normally generated line bundles on general curves, II", J. Pure Appl. Algebra 214:8 (2010), 1450-1455. MR 2011b:14014 Zbl 1185.14028

[Ein 1987] L. Ein, "A remark on the syzygies of the generic canonical curves", J. Differential Geom. 26:2 (1987), 361-365. MR 89a:14031 Zbl 0632.14024

[Eisenbud 1992] D. Eisenbud, “Green's conjecture: an orientation for algebraists”, pp. 51-78 in Free resolutions in commutative algebra and algebraic geometry (Sundance, UT, 1990), edited by D. Eisenbud and C. Huneke, Res. Notes Math. 2, Jones and Bartlett, Boston, 1992. MR 93e:13020 Zbl 0792.14015

[Eisenbud and Harris 1983] D. Eisenbud and J. Harris, "Divisors on general curves and cuspidal rational curves”, Invent. Math. 74:3 (1983), 371-418. MR 85h:14019 Zbl 0527.14022

[Eisenbud and Harris 1986] D. Eisenbud and J. Harris, "Limit linear series: basic theory", Invent. Math. 85:2 (1986), 337-371. MR 87k:14024 Zbl 0598.14003

[Farkas 2006] G. Farkas, "Syzygies of curves and the effective cone of $\bar{M}_{g}$ ", Duke Math. J. 135:1 (2006), 53-98. MR 2008a:14037 Zbl 1107.14019

[Farkas 2009] G. Farkas, "Koszul divisors on moduli spaces of curves”, Amer. J. Math. 131:3 (2009), 819-867. MR 2010f:14030 Zbl 1176.14006

[Farkas and Popa 2005] G. Farkas and M. Popa, "Effective divisors on $\bar{M}_{g}$, curves on $K 3$ surfaces, and the slope conjecture", J. Algebraic Geom. 14:2 (2005), 241-267. MR 2006a:14043 Zbl 1081.14038 
[Green 1984] M. L. Green, "Koszul cohomology and the geometry of projective varieties", J. Differential Geom. 19:1 (1984), 125-171. MR 85e:14022 Zbl 0559.14008

[Green and Lazarsfeld 1984] M. L. Green and R. Lazarsfeld, "The nonvanishing of certain Koszul cohomology groups", J. Differential Geom. 19:1 (1984), 168-170. Appendix to [Green 1984]. MR 85e:14022 Zbl 0559.14008

[Green and Lazarsfeld 1986] M. L. Green and R. Lazarsfeld, "On the projective normality of complete linear series on an algebraic curve”, Invent. Math. 83:1 (1986), 73-90. MR 87g:14022 Zbl 0594.14010

[Green and Lazarsfeld 1987] M. L. Green and R. Lazarsfeld, "Deformation theory, generic vanishing theorems, and some conjectures of Enriques, Catanese and Beauville", Invent. Math. 90:2 (1987), 389-407. MR 89b:32025 Zbl 0659.14007

[Voisin 2002] C. Voisin, "Green's generic syzygy conjecture for curves of even genus lying on a $K 3$ surface”, J. Eur. Math. Soc. (JEMS) 4:4 (2002), 363-404. MR 2003i:14040 Zbl 1080.14525

[Voisin 2005] C. Voisin, "Green's canonical syzygy conjecture for generic curves of odd genus", Compos. Math. 141:5 (2005), 1163-1190. MR 2006c:14053 Zbl 1083.14038

[Wang 2013] J. Wang, "On the projective normality of line bundles of extremal degree", Math. Ann. 355:3 (2013), 1007-1024. MR 3020151 Zbl 1269.14003

Received June 6, 2013. Revised April 14, 2014.

JIE WANG

DEPARTMENT OF MATHEMATICS

UNIVERSITY OF GEORGIA

ATHENS, GA 30602

UNITED STATES

jiewang@math.uga.edu 


\title{
PACIFIC JOURNAL OF MATHEMATICS
}

\author{
msp.org/pjm
}

Founded in 1951 by E. F. Beckenbach (1906-1982) and F. Wolf (1904-1989)

\section{EDITORS}

Don Blasius (Managing Editor)

Department of Mathematics

University of California

Los Angeles, CA 90095-1555

blasius@math.ucla.edu

\author{
Paul Balmer \\ Department of Mathematics \\ University of California \\ Los Angeles, CA 90095-1555 \\ balmer@math.ucla.edu \\ Robert Finn \\ Department of Mathematics \\ Stanford University \\ Stanford, CA 94305-2125 \\ finn@math.stanford.edu \\ Sorin Popa \\ Department of Mathematics \\ University of California \\ Los Angeles, CA 90095-1555 \\ popa@math.ucla.edu
}

\author{
Vyjayanthi Chari \\ Department of Mathematics \\ University of California \\ Riverside, CA 92521-0135 \\ chari@math.ucr.edu \\ Kefeng Liu \\ Department of Mathematics \\ University of California \\ Los Angeles, CA 90095-1555 \\ liu@math.ucla.edu \\ Jie Qing \\ Department of Mathematics \\ University of California \\ Santa Cruz, CA 95064 \\ qing@ cats.ucsc.edu
}

\section{PRODUCTION}

Silvio Levy, Scientific Editor, production@msp.org

\section{SUPPORTING INSTITUTIONS}

ACADEMIA SINICA, TAIPEI

CALIFORNIA INST. OF TECHNOLOGY

INST. DE MATEMÁTICA PURA E APLICADA

KEIO UNIVERSITY

MATH. SCIENCES RESEARCH INSTITUTE

NEW MEXICO STATE UNIV.

OREGON STATE UNIV.

\author{
STANFORD UNIVERSITY \\ UNIV. OF BRITISH COLUMBIA \\ UNIV. OF CALIFORNIA, BERKELEY \\ UNIV. OF CALIFORNIA, DAVIS \\ UNIV. OF CALIFORNIA, LOS ANGELES \\ UNIV. OF CALIFORNIA, RIVERSIDE \\ UNIV. OF CALIFORNIA, SAN DIEGO \\ UNIV. OF CALIF., SANTA BARBARA
}

\author{
Daryl Cooper \\ Department of Mathematics \\ University of California \\ Santa Barbara, CA 93106-3080 \\ cooper@math.ucsb.edu \\ Jiang-Hua Lu \\ Department of Mathematics \\ The University of Hong Kong \\ Pokfulam Rd., Hong Kong \\ jhlu@maths.hku.hk \\ Paul Yang \\ Department of Mathematics \\ Princeton University \\ Princeton NJ 08544-1000 \\ yang@math.princeton.edu
}

These supporting institutions contribute to the cost of publication of this Journal, but they are not owners or publishers and have no responsibility for its contents or policies.

See inside back cover or msp.org/pjm for submission instructions.

The subscription price for 2015 is US \$420/year for the electronic version, and \$570/year for print and electronic.

Subscriptions, requests for back issues and changes of subscribers address should be sent to Pacific Journal of Mathematics, P.O. Box 4163, Berkeley, CA 94704-0163, U.S.A. The Pacific Journal of Mathematics is indexed by Mathematical Reviews, Zentralblatt MATH, PASCAL CNRS Index, Referativnyi Zhurnal, Current Mathematical Publications and Web of Knowledge (Science Citation Index).

The Pacific Journal of Mathematics (ISSN 0030-8730) at the University of California, c/o Department of Mathematics, 798 Evans Hall \#3840, Berkeley, CA 94720-3840, is published twelve times a year. Periodical rate postage paid at Berkeley, CA 94704, and additional mailing offices. POSTMASTER: send address changes to Pacific Journal of Mathematics, P.O. Box 4163, Berkeley, CA 94704-0163.

PJM peer review and production are managed by EditFLOW ${ }^{\circledR}$ from Mathematical Sciences Publishers.

\section{PUBLISHED BY}

\section{mathematical sciences publishers \\ nonprofit scientific publishing}

http://msp.org/

(C) 2015 Mathematical Sciences Publishers 


\section{PACIFIC JOURNAL OF MATHEMATICS}

Volume $273 \quad$ No. $1 \quad$ January 2015

Maximal estimates for Schrödinger equations with inverse-square potential 1

ChangXing MiaO, JUNYong Zhang and JiQiang Zheng

Vassiliev Invariants of Virtual Legendrian Knots

PATRICIA CAHN and ASA LEVI

Some results on the generic vanishing of Koszul cohomology via

deformation theory

JIE WANG

Conformal metrics with constant curvature one and finitely many conical

singularities on compact Riemann surfaces

QING Chen, WeI WANG, Yingyi Wu and BIN XU

$\mathbb{Q}$-bases of the Néron-Severi groups of certain elliptic surfaces

MASAMICHI KURODA

On a prime zeta function of a graph

TAKehiro Hasegawa and SEIKEN SAIto

On Whittaker modules for a Lie algebra arising from the 2-dimensional torus 147

SHaObin TAN, QING WANG and ChENGKANG XU

Fréchet quantum supergroups

AXEL DE GOURSAC

Generators of the Gauss-Picard modular group in three complex dimensions

BaoHua Xie, JieYan Wang and YuePing Jiang

Complete characterization of isolated homogeneous hypersurface singularities

STEPHEN YAu and HuAiQING ZuO

A theorem of Mœglin and Waldspurger for covering groups

SHIV PRAKASH PATEL

Spanning trees and random walks on weighted graphs 